\title{
THEORY OF MONOMIAL GROUPS
}

\author{
BY \\ OYSTEIN ORE
}

The process of imbedding a group in a larger group of some prescribed type has been one of the most useful tools in the investigation of properties of groups. The three principal types of representation of groups, each with its particular field of usefulness, are the following:

1. Permutation groups.

2. Monomial groups.

3. Linear or matrix representations of groups.

These three types of representation correspond to an imbedding of the group in the following groups:

1. The symmetric group.

2. The complete monomial group.

3. The full linear group.

The symmetric group and the full linear group have both been exhaustively investigated and many of their principal properties are known. A similar study does not seem to exist for the complete monomial group. Such a general theory seems particularly desirable in view of the numerous recent investigations on finite groups in which the monomial representations are used in one form or another to obtain deep-lying theorems on the properties of such groups. The present paper is an attempt to fill this lacuna.

In this paper the monomial group or symmetry is taken in the most general sense $\left({ }^{1}\right)$ where one considers all permutations of a certain finite number of variables, each variable being multiplied also by some element of a fixed arbitrary group $H$. In the first chapter the simplest properties such as transformation, normal form, centralizer, etc., are discussed. Some of the auxiliary theorems appear to have independent interest. One finds that the symmetry contains a normal subgroup, the basis group, consisting of all those elements which do not permute the variables. The symmetry splits over the basis group with a group isomorphic to the symmetric group as one representative group. A complete solution of the problem of finding all representative groups in this splitting of the symmetry is given. This result is of interest since it gives a general idea of the solution of the splitting problem in a fairly complicated case.

In the second chapter all normal subgroups of the symmetry are deter-

Presented to the Society, February 22, 1941; received by the editors November 28, 1940.

(1) See W. Specht, Eine Verallgemeinerung der symmetrischen Gruppe, Schriften des mathematischen Seminars, Berlin, vol. 1 (1932), also W. Specht, Eine Verallgemeinerung der Permutationsgruppen, Mathematische Zeitschrift, vol. 37 (1933), pp. 321-341. 
mined. This is, as one knows, a simple problem in the case of a symmetric group. For the monomial group the problem becomes very complicated and it is found that usually a large number of normal subgroups of various types must exist. The solution depends on the determination of certain types of subgroups of direct products. The successive commutator groups of the symmetry are determined as an application $\left({ }^{2}\right)$.

The most difficult problem in this theory is the determination of all automorphisms of the symmetry. The solution given in the third chapter depends on the previous results on the splitting of the symmetry and the form of its normal subgroups. This case is different from the case of the symmetric group, for outer automorphisms will usually occur. The necessary and sufficient condition for all automorphisms to be inner automorphisms follows from the general result. These investigations are considerably complicated by several exceptions which occur in groups of the lowest orders. These cases have also been completely investigated except in the case $m=6$. Here all the necessary preparations for the calculations have been made, but since the actual solution depends on a somewhat laborious method, the work has not been carried through. It would be desirable if someone would complete the theory at this point.

The final chapter is concerned with the imbedding of an arbitrary group $G$ in a monomial group $\Sigma(H)$. Relations between the various representations are discussed and the position of the imbedded group $G$ in $\Sigma_{m}$ is investigated. Its centralizer and normalizer are determined, and the automorphisms of $G$ induced by the elements in the normalizer are obtained.

\section{Chapter I. The symmetries}

1. Definitions. Let $H$ be some group, finite or infinite. A monomial substitution over $H$ is a linear transformation

$$
\rho=\left(\begin{array}{lll}
x_{1}, & x_{2}, & \cdots, \\
r_{1} x_{i_{1}}, & r_{2} x_{i_{2}}, \cdots, r_{m} x_{i_{m}}
\end{array}\right)
$$

where each variable is changed in to some other variable multiplied by an element of $H$. We shall call the elements $r_{i}$ in (1) the factors or multipliers in $\rho$. The multiplication $r_{i} x_{j}$ is a formal one to be taken only as a pair $\left(r_{i}, x_{j}\right)$ with the associative property $r(s x)=(r s) x$.

If another such monomial substitution

$$
\kappa=\left(\begin{array}{lll}
x_{1}, & x_{2}, & \cdots, x_{m} \\
k_{1} x_{j_{1}}, & k_{2} x_{j_{2}}, \cdots, k_{m} x_{j_{m}}
\end{array}\right)
$$

is given, then the product of $\rho$ and $\kappa$ is defined by

(2) W. K. Turkin, Ueber Herstellung und Anwendungen der monomialen Darstellungen endlicher Gruppen, Mathematische Annalen, vol. 111 (1935), pp. 743-747. 


$$
\rho \kappa=\left(\begin{array}{ll}
x_{1}, & \cdots, x_{m} \\
k_{1} r_{j_{1}} x_{i_{1}}, \cdots, k_{m} r_{j_{m}} x_{i_{j_{m}}}
\end{array}\right) .
$$

The inverse of $\kappa$ is

$$
\kappa^{-1}=\left(\begin{array}{lll}
x_{j_{1}}, & \cdots, x_{j_{m}} \\
k_{1}^{-1} x_{1}, \cdots, & k_{m}^{-1} x_{m}
\end{array}\right) .
$$

By this definition of the multiplication, the set of all monomial substitutions on $m$ variables is seen to form a group $\Sigma_{m}(H)$. This group we shall call the complete monomial group of degree $m$, or somewhat more simply, the symmetry of degree $m$ of $H$. If $H$ is a finite group of order $n_{H}$ then one finds that $\Sigma_{m}(H)$ has the order $m !\left(n_{H}\right)^{m}$. The ordinary symmetric group can be considered the symmetry of the unit group.

A permutation in $\Sigma_{m}$ is a monomial substitution of the form

$$
\pi=\left(\begin{array}{l}
x_{1}, \cdots, x_{m} \\
x_{i_{1}}, \cdots, x_{i_{m}}
\end{array}\right)=\left(\begin{array}{l}
1, \cdots, m \\
i_{1}, \cdots, i_{m}
\end{array}\right) .
$$

The permutations form a subgroup $S_{m}$ of $\Sigma_{m}$ and $S_{m}$ is obviously isomorphic to the ordinary symmetric group on $m$ letters.

A monomial substitution which only multiplies each variable by a factor in $H$

$$
\mu=\left(\begin{array}{l}
x_{1}, \cdots, x_{m} \\
r_{1} x_{1}, \cdots, r_{m} x_{m}
\end{array}\right)=\left[r_{1}, \cdots, r_{m}\right]
$$

will be called a multiplication. The multiplications $\mu$ form a subgroup $V_{m}(H)$ of $\Sigma_{m}(H)$ which we shall call the basis group. It is easily seen that the basis group is a normal subgroup of $\Sigma_{m}(H)$. Furthermore it is the direct product of $m$ groups isomorphic to $H$

$$
V_{m}=H_{1}^{*} \times \cdots \times H_{m}^{*}
$$

where $H_{i}^{*}$ consists of the multiplications

$$
\eta^{(i)}=\left[1, \cdots, 1, r_{i}, 1, \cdots, 1\right]
$$

where $r_{i}$ runs through $H$.

The special multiplications

$$
\mu=[a, a, \cdots, a]=[a]
$$

where all factors are equal, will be called scalars. The scalars are the only elements which commute with all permutations. The center of the symmetry consists of all scalars

$$
\sigma=[c, c, \cdots, c]
$$


where $c$ belongs to the center of $H$. This shows that the centers of $H$ and $\Sigma_{m}(H)$ are isomorphic groups.

Any monomial substitution can be written uniquely as the product of a permutation and a multiplication. If $\rho, \pi$ and $\mu$ are given by (1), (4) and (5), respectively, one finds $\rho=\pi \mu$. This shows that one has

$$
\Sigma_{m}=S_{m} \cup V_{m}, \quad S_{m} \cap V_{m}=E .
$$

2. Auxiliary results. Before we proceed to a more detailed study of the symmetries, it is necessary to derive a few auxiliary theorems which also have some independent interest.

We first prove the following theorem:

THEOREM 1. Let $d_{1}, d_{2}, \cdots, d_{n}$ be $n$ elements of a group $H$ all belonging to the same class. Then there exist elements $c_{i}$ in $H$ such that

$$
d_{1}=c_{1} \cdots c_{n}, d_{2}=c_{2} \cdots c_{n} c_{1}, \cdots, d_{n}=c_{n} c_{1} \cdots c_{n-1} .
$$

Proof. According to the assumption we may write

$$
d_{2}=c_{1}^{-1} d_{1} c_{1}, d_{3}=c_{2}^{-1} d_{2} c_{2}, \cdots, d_{n}=c_{n-1}^{-1} d_{n-1} c_{n-1} .
$$

Then is is only necessary to determine the element $c_{n}$ such that $d_{1}=c_{1} \cdots c_{n-1} c_{n}$ and one finds that all other relations (9) are satisfied.

For $n=2$ the theorem expresses the fact that if $\dot{d}_{1}$ and $d_{2}$ belong to the same class then there exist elements $c_{1}$ and $c_{2}$ in $H$ such that $d_{1}=c_{1} c_{2}, d_{2}=c_{2} c_{1}$.

Next we prove:

THEOREM 2. Let the products $a_{1} a_{2} \cdots a_{n}, b_{1} b_{2} \cdots b_{n}$ belong to the same class in a group $H$. Then there exist elements $q_{1}, \cdots, q_{n}$ in $H$ such that

$$
b_{1}=q_{1}^{-1} a_{1} q_{2}, b_{2}=q_{2}^{-1} a_{2} q_{3}, \cdots, b_{n}=q_{n}^{-1} a_{n} q_{1} .
$$

Proof. If one writes $b_{1} \cdots b_{n}=q_{1}^{-1}\left(a_{1} \cdots a_{n}\right) q_{1}$ then one finds that the relations (10) can be satisfied by putting

$$
q_{i}=a_{i-1}^{-1} \cdots a_{1}^{-1} q_{1} b_{1} \cdots b_{i-1} \text {. }
$$

3. Cycles and transformations. In the theory of monomial groups as well as in the theory of substitution groups it is advantageous to introduce cycles of monomial substitutions, i.e., substitutions having the form

$$
\gamma=\left(\begin{array}{ll}
x_{1}, & x_{2}, \cdots, x_{n} \\
c_{1} x_{2}, & c_{2} x_{3}, \cdots, c_{n} x_{1}
\end{array}\right)=\left(c_{1} x_{2}, c_{2} x_{3}, \cdots, c_{n-1} x_{n}, c_{n} x_{1}\right) .
$$

As in the theory of permutation groups one shows:

THEOREM 3. A monomial substitution can be written uniquely as the product of commutative cycles without common variables. 
One finds that the $n$th power of a cycle (11) of length $n$ is a multiplication

$$
\gamma^{n}=\left(\begin{array}{l}
x_{1}, \cdots, x_{n} \\
\Delta_{1} x_{1}, \cdots, \Delta_{n} x_{n}
\end{array}\right)=\left[\Delta_{1}, \Delta_{2}, \cdots, \Delta_{n}\right] .
$$

Here the factors $\Delta_{i}$ are determined by

$$
\Delta_{1}=c_{1} c_{2} \cdots c_{n}, \Delta_{2}=c_{2} \cdots c_{n} c_{1}, \cdots, \Delta_{n}=c_{n} c_{1} \cdots c_{n-1} .
$$

These elements in $H$ will be called the determinants of $\gamma$. Since one can write the relations (13) in the form

$$
\Delta_{2}=c_{1}^{-1} \Delta_{1} c_{1}, \cdots, \Delta_{n}=c_{n-1}^{-1} \Delta_{n-1} c_{n-1}, \Delta_{1}=c_{n}^{-1} \Delta_{n} c_{n},
$$

one concludes that with each cycle (11) there is associated a unique determinant class in $H$.

From (12) one concludes:

THEOREM 4. The order of a cycle $\gamma$ of length $n$ is $n t$ where $t$ is the order of its determinant class. The order of any monomial substitution is the least common multiple of the orders of its cycles.

We shall next prove the following fact.

TheOREM 5. Any elements $d_{1}, \cdots, d_{n}$ in the same class in $H$ are the determinants of some monomial substitution of length $n$.

Proof. This theorem is a restatement of Theorem 1.

We shall now turn to the transformation of one monomial substitution by another. Obviously it is sufficient to study what happens to a single cycle (11) by transformation. From (2) and (3) one obtains

$$
\kappa \gamma \kappa^{-1}=\left(\begin{array}{lll}
x_{j_{1}}, & x_{j_{2}}, & \cdots, \\
k_{1}^{-1} c_{1} k_{2} x_{j_{2}}, & k_{2}^{-1} c_{2} k_{3} x_{j_{3}}, \cdots, k_{n}^{-1} c_{n} k_{1} x_{j_{1}}
\end{array}\right) .
$$

This shows that every cycle goes into a cycle of the same length. Furthermore the determinants of the transformed cycle (15) are similar to those of $\gamma$, namely

$$
\Delta_{1}^{\prime}=k_{1}^{-1} \Delta_{1} k_{1}, \cdots, \Delta_{n}^{\prime}=k_{n}^{-1} \Delta_{n} k_{n} .
$$

This proves that the determinant class of a cycle is invariant under transformation.

THEOREM 6. The necessary and sufficient condition for two monomial cycles to be similar is that they shall have the same length and the same determinant class.

Proof. It has already been shown that the condition is necessary and the sufficiency follows from (15) and Theorem 2.

From Theorem 6 one draws the more general conclusion that two mono- 
mial substitutions are similar if and only if the cycles in their cyclic decompositions may be made to correspond in such a manner that corresponding cycles have the same length and determinant class.

We show next:

ThEOREM 7. Any cycle of length $n$ may be transformed to the normal form

$$
\gamma=\left(\begin{array}{l}
x_{i_{1}}, \cdots, x_{i_{n-1}}, x_{i_{n}} \\
x_{i_{2}}, \cdots, x_{i_{n}}, a x_{i_{1}}
\end{array}\right)=\left(x_{i_{2}}, \cdots, x_{i_{n}}, a . x_{i_{1}}\right)
$$

where $a$ is any element in the determinant class of $\gamma$. Any monomial substitution $\rho$ is similar to a product of cycles without common variables $\rho=\gamma_{1} \cdots \gamma_{r}$ where each cycle is in normal form.

Proof. The determinant class of the cycle (17) is the same as the class of $a$, and hence a cycle may be transformed into the form (17) by Theorem 6 . One sees also directly from (15) that a cycle may be transformed in such a way that the $n-1$ first factors become the unit element. Since the transformation of $\gamma$ into the form (17) may be performed by means of a substitution involving only the same variables, all cycles in $\rho$ may be transformed into normal form simultaneously.

4. Centralizers. We shall use the preceding results to determine the centralizer of an arbitrary element $\rho$ in the symmetry. Since similar elements have similar centralizers one can assume that $\rho$ is in the normal form of Theorem 7.

The centralizer shall be determined by the investigation of a few special cases. We assume first that $\rho=\gamma_{a}$ is a single cycle

$$
\gamma_{a}=\left(a x_{1}, x_{2}, \cdots, x_{n}\right) .
$$

When $\gamma_{a}$ is transformed by $\kappa$ in (2) one finds as in (15)

$$
\kappa \gamma_{a} \kappa^{-1}=\left(k_{n}^{-1} a k_{1} x_{j_{1}}, k_{1}^{-1} k_{2} x_{j_{2}}, \cdots, k_{n-1}^{-1} k_{n} x_{j_{n}}\right) .
$$

Since this cycle will be identical with (18) when $\kappa$ belongs to the centralizer one sees first that $\kappa$ must have the form

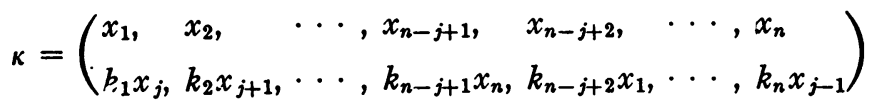

while the substitution on the remaining $m-n$ variables is immaterial. For this reason we consider only the centralizer $C_{\gamma}$ of $\gamma$ in $\Sigma_{n}(H)$. The values of the factors $k_{i}$ in $\kappa$ can be obtained by comparison of (18) and (19) and one finds without difficulty that an arbitrary element $\kappa$ in the centralizer $C_{\gamma}$ has the form 


$$
\kappa=\left(\begin{array}{l}
x_{1}, \cdots, x_{n-j+1}, x_{n-j+2}, \cdots, x_{n} \\
c x_{j}, \cdots, c x_{n}, \quad c a x_{1}, \cdots, c a x_{j-1}
\end{array}\right)
$$

where $c$ is an arbitrary element in the centralizer of $a$ in $H$. Obviously the powers of the cycle $\gamma_{a}$ must belong to $C_{\gamma}$ and one sees directly that

$$
\kappa=[c] \gamma_{a}^{j}=\gamma_{a}^{j}[c]
$$

where $[c]$ is the multiplication in which every variable is multiplied by $c$. This shows that the centralizer $C_{\gamma}$ is isomorphic to a cyclic extension of degree $n$ of a group isomorphic to the centralizer of $a$ in $H$. If this last centralizer is finite and of order $r_{a}$ then one sees that the order of $C_{\gamma}$ is $n r_{a}$.

Next let us determine the centralizer $C_{\lambda}$ of a product of cycles of the same length $n$ and the same determinant class $a$,

$$
\lambda=\gamma^{(1)} \cdots \gamma^{(k)},
$$

in the symmetry of degree corresponding to the variables involved. Obviously any permutation of the cycles $\gamma^{(i)}$ in (20) among themselves must belong to the centralizer $C_{\lambda}$, and an element of the centralizer leaving all cycles in $\rho$ fixed by transformation must have the form

$$
c_{\lambda}=c^{(1)} \cdots c^{(k)}
$$

where $c^{(i)}$ belongs to the centralizer of $\gamma^{(i)}$ in the symmetry on its variables. From these results one concludes that $C_{\lambda}$ is isomorphic to the symmetry $\Sigma_{k}\left(C_{\gamma}\right)$, where $C_{\gamma}$ is the centralizer of a single cycle $\gamma$.

Since any element $\rho$ in $\Sigma_{m}(H)$ can be written as a product of cycles $\gamma$ when it is transformed to normal form, one finally obtains:

THEOREM 8. Let an element in $\Sigma_{m}(H)$ be transformed into its normal form

$$
\rho=\lambda_{1} \cdots \lambda_{l}, \quad \lambda_{i}=\gamma_{1}^{(i)} \cdots \gamma_{k_{i}}^{(i)}
$$

where for a fixed $i$ the $\gamma_{j}^{(i)}$ are the normalized cycles of the same length $n_{i}$ and the same determinant class $a_{i}$. Then the centralizer $C_{\rho}$ of $\rho$ in $\Sigma_{m}(H)$ is isomorphic to the direct product of symmetries

$$
C_{\rho} \simeq \Sigma_{k_{1}}\left(C_{a_{1}}\right) \times \Sigma_{k_{2}}\left(C_{a_{2}}\right) \times \cdots
$$

where $C_{a_{i}}$ is the centralizer of a single cycle $\gamma_{j}^{(i)}$ in $\Sigma_{n_{i}}(H)$. The group $C_{a_{i}}$ consists of all elements of the form $\kappa=\left[c_{i}\right] \gamma_{1}^{(i)}$, where the element $c_{i}$ belongs to the normalizer of $a_{i}$ in $H$.

Again if $r_{i}$ is the order of the centralizer of $a_{i}$ in $H$ one finds that the order of $C_{\rho}$ is

$$
N=\prod k_{i} !\left(n_{i} r_{i}\right)^{k_{i}}
$$


5. Splitting of the symmetry. A group $G$ containing a normal subgroup $N$ is said to split over $N$ if there exists a subgroup. $M$ such that

$$
G=M \cup N, \quad M \cap N=E .
$$

In this representation the group $M$ can be replaced by any of its conjugates and the relations will still hold. There may, however, exist other groups $M_{1}$ such that

$$
G=M_{1} \cup N, \quad M_{1} \cap N=E
$$

and such that $M_{1}$ is not a conjugate of $M$. This leads to a division of all representative groups $M$ in to classes, each consisting of conjugate groups. When there is only one class, hence when all $M$ are conjugate, we say that $G$ splits regularly over $N$.

We have already seen in (8) that for the symmetry one has

$$
\Sigma_{n}=S_{m} \cup V_{m}, \quad S_{m} \cap V_{m}=E
$$

and hence $\Sigma_{m}$ splits over $V_{m}$. We shall now consider the problem of finding all groups $T$ such that

$$
\Sigma_{m}=T \cup V_{m}, \quad T \cap V_{m}=E .
$$

One sees immediately that $T$ is a group isomorphic to $S_{m}$ and the isomorphism is such that each element in $T$ is obtained from the corresponding element in $S_{m}$ by multiplication with an element in the basis group $V_{m}$.

The group $S_{m}$ contains the transpositions $(1, i)$. This implies that $T$ contains $m-1$ substitutions of the form

$$
\lambda_{i}=\left[a_{1, i}, \cdots, a_{m, i}\right]\left(x_{1}, x_{i}\right) .
$$

Let us transform all $\lambda_{i}$ by the multiplication

$$
\kappa=\left[k_{1}, \cdots, k_{m}\right] .
$$

One finds that the group $\kappa T \kappa^{-1}$ contains all the substitutions

$$
\lambda_{i}^{\prime}=\left[k_{i}^{-1} a_{1, i} k_{1}, \cdots, k_{1}^{-1} a_{i, i} k_{i}, \cdots, k_{j}^{-1} a_{j, i} k_{j}, \cdots\right]\left(x_{1}, x_{i}\right)
$$

where $j$ is an index different from 1 and $i$. This shows that it is possible to choose $\kappa$ in such a manner that $a_{1, i}=1$ for every $i$, and in the following we shall make this assumption.

Next one finds

$$
\lambda_{i}^{2}=\left[a_{i, i}, a_{2, i}^{2}, \cdots, a_{i, i}, \cdots, a_{m, i}^{2}\right]
$$

and since it is a multiplication contained in $T$ one must have $\lambda_{i}^{2}=E$; hence

$$
a_{i, i}=a_{1, i}=1, \quad a_{i, j}^{2}=1 .
$$


In the special case $m=2$ it follows already from these conditions that $\lambda_{2}=(1,2)$ and we have shown:

TheOREM 9. For $m=2$ the symmetry splits regularly over its basis group.

In the following we can assume $m \geqq 3$. Let us form the product

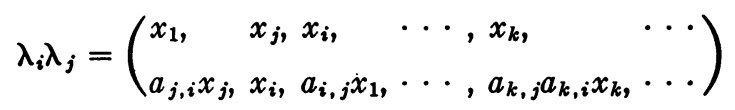

where $k$ is any index different from $1, i$ and $j$. Since the third power of this substitution must also be the unit substitution one obtains the conditions

$$
a_{j, i} a_{i, j}=1 . \quad\left(a_{k, j} a_{k, i}\right)^{3}=1, \quad k \neq i, k \neq j .
$$

When the first of these conditions is combined with (24) one obtains

$$
a_{i, j}=a_{j, i}
$$

The results expressed in (23) and (25) are already sufficient to give a complete solution for the case $m=3$. One finds then

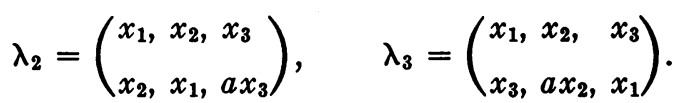

These two elements generate a group isomorphic to $S_{3}$ consisting of the substitutions (26), the unit element and the following three substitutions:

$$
\begin{aligned}
& \mu=\left(\begin{array}{ccc}
x_{1}, & x_{2}, & x_{3} \\
a x_{3}, & a x_{1}, & x_{2}
\end{array}\right), \quad \mu^{2}=\left(\begin{array}{ccc}
x_{1}, & x_{2}, & x_{3} \\
a x_{2}, & x_{3}, & a x_{1}
\end{array}\right), \\
& \nu=\left(\begin{array}{lll}
x_{1}, & x_{2}, & x_{3} \\
a x_{1}, & a x_{3}, & a x_{2}
\end{array}\right) \text {. }
\end{aligned}
$$

From now on we can even assume $m \geqq 4$. We evaluate the product

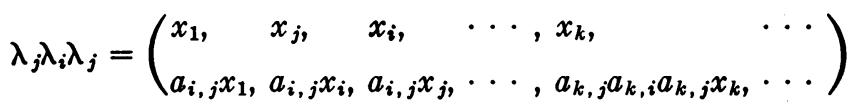

where $k$ denotes any index different from $1, i, j$. In the isomorphism between $S_{m}$ and $T$ the substitution $\lambda_{i}$ corresponds to the transposition $(1, i)$ in $S_{m}$, and hence $\lambda_{j} \lambda_{i} \lambda_{j}$ must correspond to $(i, j)$.

Next we utilize the fact that in $S_{m}$

$$
(1, h)(i, j)=(i, j)(1, h)
$$

for four different indices $1, h, i, j$. This implies for the corresponding elements in $T$

$$
\lambda_{h} \cdot \lambda_{j} \lambda_{i} \lambda_{j}=\lambda_{j} \lambda_{i} \lambda_{j} \cdot \lambda_{h}
$$


When these products are compared one finds that they imply the identities

$$
a_{i, j}=a_{h, j} a_{h, i} a_{h, j}, \quad a_{i, j} a_{k, h}=a_{k, h} a_{i, j} .
$$

When the first of these relations is applied to (28) one finds the simpler expression

$$
\lambda_{j} \lambda_{i} \lambda_{j}=\left[a_{i, j}\right]\left(\begin{array}{ll}
x_{i}, & x_{j} \\
x_{j}, & x_{i}
\end{array}\right) .
$$

This important relation shows that if $i \neq 1, j \neq 1$, then the element in $T$ corresponding to $(i, j)$ in $S_{m}$ is obtained simply through the multiplication of $(i, j)$ by a scalar $\left[a_{i, j}\right]$. But the transpositions $(i, j)$ generate the group of all permutations $\pi_{1}$ leaving the element 1 fixed; hence one has for any such permutation

$$
\pi_{1} \rightarrow \pi_{1}\left[a_{\pi_{1}}\right] .
$$

Since the scalars commute with all permutations this shows that the $a_{\pi_{1}}$ form a subgroup of $H$ which is homomorphic to the symmetric group on $m-1$ letters.

This leads to the following theorem:

THEOREM 10. The symmetry $\Sigma_{m}(H)$ splits over its basis group $\Sigma_{m}=T \cup V_{m}$, $T \cap V_{m}=E$. Any group $T$ in this decomposition is the conjugate of some group $T_{0}$ obtained by the following construction. Let $A$ be any subgroup of $H$ homomorphic to the symmetric group on $m-1$ letters, and let $\pi_{1} \rightarrow a_{\pi_{1}}$ indicate the homomorphism between $A$ and the permutations $\pi_{1}$ in the symmetric group $S_{m-1}^{(1)}$ on the $m-1$ letters $2, \cdots, m$. In particular we shall write

$$
(i, j) \rightarrow a_{i, j}, \quad(i, i) \rightarrow 1=a_{i, i} .
$$

Then the elements of $T_{0}$ are obtained from the permutations of the symmetric group $S_{m}$ by the isomorphism

$$
\begin{gathered}
\pi_{1} \rightarrow \pi_{1}\left[a_{\pi_{1}}\right] \text { for } \pi_{1} \text { in } S_{m-1}^{(1)}, \\
(1, i) \rightarrow \lambda_{\imath}=\left[1, a_{2, i}, \cdots, a_{m, i}\right](1, i) .
\end{gathered}
$$

It has already been shown that any group $T$ after suitable transformation must have the form indicated by the correspondence (30). It remains to show that the set of substitutions defined by the correspondence (30) actually forms a group isomorphic to $S_{m}$.

We observe first that any permutation in $S_{m}$ can be written uniquely in the form

$$
\pi=(1, i) \pi_{1}
$$

where $\pi_{1}$ leaves 1 fixed. Thus (30) defines a unique substitution $T_{x}$ corresponding to each permutation $\pi$, namely, 


$$
T_{\pi}=T_{(1, i)} \cdot T_{\pi_{1}}
$$

or

$$
T_{\pi}=\left[1, a_{2, i}, \cdots, a_{m, i}\right]\left[a_{\pi_{1}}\right](1, i) \pi_{1}
$$

and therefore

$$
T_{\pi}=\left[a_{\pi_{1}}, a_{2, i} a_{\pi_{1}}, \cdots, a_{m, i} a_{\pi_{1}}\right] \pi .
$$

Our problem is to prove that this correspondence is an isomorphism. Let $\rho=(1, j) \rho_{1}$ be another permutation in the normal form (31) where $\rho_{1}$ leaves 1 fixed. We shall then have to prove

$$
T_{\pi} \cdot T_{\rho}=T_{\pi \rho}
$$

From the definition (33) of $T_{\pi}$ it follows that

$$
T_{\pi \rho_{1}}=T_{\pi} \cdot T_{\rho_{1}}
$$

for any $\pi$ and any $\rho_{1}$ leaving 1 fixed. When this is applied to (34) one sees that it is no limitation to assume simply $\rho=(1, j)$.

We shall have to prove, therefore, that

$$
T_{x(1, j)}=T_{\pi} \cdot T_{(1, j)}=T_{(1, i)} \cdot T_{\pi_{1}} \cdot T_{(1, j)}
$$

and this follows if one can prove the two simpler relations

$$
T_{(1, i)} \cdot T_{\rho}=T_{(1, i) \rho}
$$

for any permutation $\rho$, and

$$
T_{\pi_{1} \rho}=T_{\pi_{1}} \cdot T_{\rho}
$$

for any $\pi_{1}$ leaving 1 fixed.

Let us first turn our attention to the relation (35). By the preceding remark it is sufficient to prove

$$
T_{(1, i)} \cdot T_{(1, j)}=T_{(1, i)(1, j)} .
$$

For $i=j$ this relation follows simply from $a_{i, j}^{2}=1$. We assume therefore $i \neq j$ and find

$$
T_{(1, i)} \cdot T_{(1, j)}=\left[1, \cdots, a_{t, i}, \cdots\right](1, i)\left[1, \cdots, a_{t, j}, \cdots\right](1, j)
$$

or

$$
T_{(1, i)} \cdot T_{(1, j)}=\left(\begin{array}{ll}
x_{1}, & x_{i}, \cdots, x_{t}, \\
a_{i, j} x_{1}, & x_{i}, \cdots, a_{t, j} a_{t, i} x_{t}, \cdots
\end{array}\right)(1, i)(1, j)
$$

where $t$ is any index different from 1 and $i$. On the other hand one obtains

$$
T_{(1, i)(1, j)}=T_{(1, j, i)}=T_{(1, j)(i, j)}=\left[1, \cdots, a_{t, j}, \cdots\right]\left[a_{i, j}\right](1, i)(1, j)
$$


or

$$
T_{(1, i)(1, j)}=\left(\begin{array}{lll}
x_{1}, & x_{i}, \cdots, x_{t}, & \cdots \\
a_{i, j} x_{1}, & x_{i}, \cdots, a_{i, j} a_{t, j} x_{i}, \cdots
\end{array}\right)(1, i)(1, j) .
$$

Since $a_{i, j}=a_{i, i}, a_{t, j} a_{t, i}=a_{i, j} a_{t, j}$ the relation (37) follows.

To prove the relation (36) let us observe that $\pi$ can be written as the product of transpositions not involving 1 . If (36) is proved for any transposition $\pi_{1}$ it will therefore follow in general. By the preceding remarks we can also assume $\rho=(1, j)$, and hence the proof of $(36)$ reduces to

$$
T_{(k, l)} \cdot T_{(1, j)}=T_{(k, l)(1, j)} \text {. }
$$

Here we may assume $k \neq l$ and one needs only to study the two cases $j \neq l$ and $j=l$ since $k$ and $l$ occur symmetrically. A simple calculation which we shall not reproduce here shows that (38) actually holds in both cases.

This concludes the proof of Theorem 10. It should be observed that this theorem is of interest since it gives complete information about a splitting extension in a general and rather involved case.

To conclude let us prove:

THEOREM 11. The necessary and sufficient condition for the symmetry $\Sigma_{m}(H)$ to split regularly over its basis group $V_{m}(H)$ is that $H$ contain no subgroup different from $E$ homomorphic to the symmetric group on $m-1$ letters.

Proof. If $H$ contains no such group, all the factors $a_{i, j}$ in Theorem 10 are the unit element and the group constructed is the symmetric group $S_{m}$.

Conversely if $\Sigma_{m}$ splits regularly the group $T$ can be transformed into $S_{m}$. Since the transformation of a substitution in $\Sigma_{m}$ by a permutation only permutes its factors it follows that this transformation is a multiplication,

$$
\kappa=\left[k_{1}, \cdots, k_{m}\right] .
$$

But when $\kappa$ transforms

$$
\lambda_{i}=\left[1, a_{2, i}, \cdots, a_{m, i}\right](1, i)
$$

in to a permutation one finds that all $k_{i}$ are equal and $a_{i, j}=1$.

\section{Chapter II. Normal subgroups of the Symmetry}

1. Permutation-invariant subgroups. The main problem to be considered in the following is the determination of all normal subgroups of the symmetry $\Sigma_{m}(H)$. Before this problem can be solved it is however necessary to solve a preliminary problem which also has some independent interest.

We shall say that a subgroup of the symmetry is permutation invariant if it is transformed into itself by all permutations $\pi$ of the symmetric group $S_{m}$. The first problem to be considered is then the determination of all permutation invariant subgroups of the basis group $V_{m}$. 
Let $\bar{N}$ be a permutation invariant subgroup of $V_{m}$,

$$
\kappa=\left[k_{1}, \cdots, k_{m}\right]
$$

one of its elements and

$$
\pi=\left(\begin{array}{l}
1, \cdots, m \\
i_{1}, \cdots, i_{m}
\end{array}\right)
$$

an arbitrary permutation. Since $\bar{N}$ is permutation invariant it must also contain all multiplications

$$
\kappa_{\mu}=\pi^{-1} \kappa \pi=\left[k_{i_{1}}, \cdots, k_{i_{m}}\right]
$$

and conversely if $\bar{N}$ contains all multiplications (3) when it contains $\kappa, \bar{N}$ is permutation invariant. One sees easily that if $\bar{N}$ and $\bar{M}$ are permutation invariant subgroups of $V_{m}$ then $\bar{N} \cup \bar{M}$ and $\bar{N} \cap \bar{M}$ have the same property, and hence the permutation invariant subgroups of $V_{m}$ form a structure.

Let us mention three simple types of permutation invariant subgroups of $V_{m}$.

1. Subgroups $\bar{N}$ where the $k_{i}$ in (1) run through all elements in a certain subgroup $K$ of $H$ independently. Then $\bar{N}$ is isomorphic to the direct product of $m$ groups, each isomorphic to $K$.

2. The $k_{i}$ run through $K$ in such a manner that $k_{1}=k_{2}=\cdots=k_{m}$. In this case $\bar{N}$ is isomorphic to $K$.

3. $K$ is Abelian and the $k_{i}$ are so restricted that $k_{1} k_{2} \cdots k_{m}=e$. In this case $\bar{N}$ is isomorphic to the direct product of $m-1$ factors, each isomorphic to $K$.

In the following we shall show that all permutation invariant subgroups of $V_{m}$ may be obtained essentially through a combination of these three types.

The proposed problem may also be formulated in a slightly different manner. We have already seen that the basis group $V_{m}$ is isomorphic to the direct product

$$
V_{m} \cong H^{(1)} \times \cdots \times H^{(m)}
$$

where each group $H^{(i)}$ is isomorphic to $H$. Now conversely let $m$ such groups $H^{(i)}$ be given and let $T_{i}$ denote the isomorphism which takes $H$ into $H^{(i)}$. Then the direct product $P=H^{(1)} \times \cdots \times H^{(m)}$ consists of all elements of the form

$$
p=h_{1}^{T_{1}} h_{2}^{T_{2}} \cdots h_{m}^{T_{m}} .
$$

Our problem is then equivalent to the determination of all those subgroups of $P$ which have the property that when (4) is contained in the subgroup, then it also contains all the products $p^{\prime}=h_{i_{1}}^{T_{1}} h_{i_{2}}^{T_{2}} \cdots h_{i_{m}}^{T_{m}}$ obtained by an arbitrary permutation of the indices. 
2. Permutation invariant subgroups for $m=2$. From now on let $\bar{N}$ denote a fixed permutation invariant subgroup of the basis group $V_{m}$ and let $\kappa$ in (1) be one of its elements. All elements $k_{i}$ of $H$ which occur as the $i$ th factor in any such multiplication will form a subgroup $K_{i}$ of $H$. But since $\bar{N}$ is permutation invariant this group must be the same, say $K$, for all indices $i$. One sees then that in order to determine $\bar{N}$ it is necessary to know $K$ and the rule which determines which elements $k_{i}$ in $K$ may be combined to give an element (1) of $\bar{N}$.

There exist certain subgroups of the permutation invariant subgroup $\bar{N}$ of $V_{m}$ which are of particular importance. Those elements of $\bar{N}$ which have the form

$$
\sigma_{i}=\left[s_{1}, \cdots, s_{i}, 1, \cdots, 1\right]
$$

form a subgroup $\bar{S}_{i}$ of $\bar{N}$. When $\sigma_{i}$ is transformed by $\kappa$ in (1) one finds

$$
\kappa \sigma_{i} \kappa^{-1}=\left[k_{1}^{-1} s_{1} k_{1}, \cdots, k_{i}^{-1} s_{i} k_{i}, 1, \cdots, 1\right] .
$$

This shows that $\bar{S}_{i}$ is normal in $\bar{N}$. Furthermore those $s_{j}$ in (5) which occur as factors at the $j$ th place also form a subgroup $S_{i}$ of $H$ independent of $j$ and $S_{i}$ is normal in $K$.

When the various permutations $\pi$ in (2) are applied to $\bar{S}_{i}$ one obtains $C_{m, i}$ other subgroups $\bar{S}_{i}^{(\pi)}$ leaving $i$ variables unchanged. Among these groups we shall consider first the $m$ groups $\bar{S}_{1}^{(j)}(j=1,2, \cdots, m)$ consisting of the elements of the form

$$
\left[1, \cdots, 1, s_{j}, 1, \cdots, 1\right] .
$$

We have already seen that the corresponding $s_{j}$ form a normal subgroup $S_{1}$ of $K$. But then the direct product

$$
P_{1}=\bar{S}_{1}^{(1)} \times \cdots \times \bar{S}_{1}^{(m)}
$$

is a normal subgroup of $\bar{N}$. Furthermore $P_{1}$ is permutation invariant of the first type indicated in $\S 1$ and obviously it is the largest such group contained in $\bar{N}$. This means that if $\kappa$ in (1) is an arbitrary element of $\bar{N}$ then any $k_{i}$ may be multiplied by an arbitrary $s_{i}$ in $S_{1}$ and still $\kappa$ remains in $\bar{N}$. This shows that the eventual conditions on the relations between the various $k_{i}$ in an element (1) in $\bar{N}$ can only be conditions mod $S_{1}$. It is therefore no limitation if we consider the quotient group $K / S_{1}$ in the following and therefore assume $S_{1}=1$; hence $s_{j}=1$ in (6).

The case $m=2$ presents a certain exception and we shall therefore solve our problem first in this case. If $\bar{N}$ contains two elements

$$
\alpha=\left[a_{1}, a_{2}\right], \quad \beta=\left[a_{1}, b_{2}\right]
$$

with the same first component $a_{1}$ then 


$$
\alpha \beta^{-1}=\left[1, b_{2}^{-1} a_{2}\right]=[1,1]
$$

and hence $a_{2}=b_{2}$. This shows that $a_{1}$ determines $a_{2}$ uniquely and conversely. Furthermore the correspondence between $a_{1}$ and $a_{2}$ is an automorphism $T$ of $K: a_{2}=a_{1}^{T}$. But since $\bar{N}$ is permutation invariant, $a_{1}$ and $a_{2}$ may be interchanged and one finds $a_{1}=a_{2}^{T}$, and $T$ is an automorphism of order 2. Conversely one finds that the multiplications $\left[a_{1}, a_{1}^{T}\right]$ do form a permutation invariant subgroup of $V_{2}$ if $T$ is an automorphism of $K$ of order 2 . We have therefore shown:

THEOREM 1. All permutation invariant subgroups $\bar{N}$ of $V_{2}(H)$ can be obtained by the following construction. A subgroup $K$ of $H$ is chosen. $S_{1}$ denotes an arbitrary normal subgroup of $K$ while $T$ is a fixed automorphism of order 2 of the quotient group $K / S_{1}$. Then $\bar{N}$ consists of all elements

$$
\alpha=\left[a_{1}, a_{2}\right]
$$

where $a_{1}$ and $a_{2}$ run through $K$ in such a manner that

$$
a_{2} \equiv a_{1}^{T}\left(\bmod S_{1}\right) \text {. }
$$

3. Permutation invariant subgroups of the basis group in the general case. We shall now turn to the determination of the permutation invariant subgroups $\bar{N}$ of $V_{m}(H)$ in the general case where $m \geqq 3$. Let us recall also that we have assumed $S_{1}=1$. We shall first study in some detail the properties of the group $\bar{S}_{2}$ which was defined in $\S 2$ as the set of all elements of $\bar{N}$ of the form $\alpha_{2}=\left[s_{1}, s_{2}, 1, \cdots, 1\right]$. Since $\bar{N}$ is permutation invariant, $\bar{S}_{2}$ must be unchanged when $s_{1}$ and $s_{2}$ are interchanged. Theorem 1 may therefore be applied and one concludes that

$$
\alpha_{2}=\left[s_{1}, \underset{s_{1}}{T}, 1, \cdots, 1\right]
$$

where $T$ is some automorphism of order 2 of the group $S_{2}$.

When $\alpha_{2}$ in ( 7$)$ is transformed by an arbitrary permutation one finds that for any element

$$
\left[1, \cdots, 1, s_{i}, 1, \cdots, 1, s_{j}, 1, \cdots, 1\right]
$$

in $\bar{N}$ one has $s_{j}=s_{i}^{T}$. But when $\bar{N}$ contains ( $(\bar{\tau})$ it also contains

$$
\beta_{2}=\left[s_{1}, 1, \stackrel{T}{s_{1}}, 1, \cdots, 1\right]
$$

and hence the quotient

$$
\alpha_{2} \beta_{2}^{-1}=\left[1, s_{1}^{T},\left(s_{1}^{-1}\right)^{T}, 1, \cdots, 1\right],
$$

and one finds

$$
\stackrel{T}{s_{1}}=s_{1}^{-1}
$$


But if a group has an automorphism changing each element into its inverse then $\left(s_{1} s_{2}\right)^{T}=\left(s_{1} s_{2}\right)^{-1}=s_{1}^{T} s_{2}^{T}=s_{1}^{-1} s_{2}^{-1}$ or $s_{2} s_{1}=s_{1} s_{2}$ and the group is Abelian. We have therefore obtained the following result:

The group $\bar{S}_{2}$ consists of all elements

$$
\alpha_{2}=\left[s_{1}, s_{1}^{-1}, 1, \cdots, 1\right]
$$

where $s_{1}$ runs through a normal Abelian subgroup of $K$.

When the elements $s_{1}$ and $s_{1}^{-1}$ in (8) are permuted into all possible positions, the corresponding substitutions generate a normal subgroup $\bar{R}$ of $\bar{N}$ which is also permutation invariant. From the construction of this group it follows that if $\rho=\left[r_{1}, \cdots, r_{m}\right]$ is one of its elements, then

$$
r_{1} r_{2} \cdots r_{m}=1,
$$

and hence $\bar{R}$ is a group of the third type indicated in $\S 1$. From the definition of $\bar{R}$ it follows that it contains all substitutions

$$
\begin{aligned}
& \rho_{1}=\left[p_{1}, p_{1}^{-1}, 1, \cdots, 1\right], \quad \rho_{2}=\left[1, p_{2}, p_{2}^{-1}, 1, \cdots, 1\right], \\
& \rho_{m-1}=\left[1, \cdots, 1, p_{m-1}, p_{m-1}^{-1}\right],
\end{aligned}
$$

where the $p_{i}$ are arbitrary elements in $S_{2}$. But then $\bar{R}$ contains the product

$$
\gamma=\rho_{1} \rho_{2} \cdots \rho_{m-1}
$$

or

$$
\gamma=\left[p_{1}, p_{2} p_{1}^{-1}, \cdots, p_{m-1} p_{m-2}^{-1}, p_{m-1}^{-1}\right]
$$

and one sees easily that every element in $\bar{R}$ can be expressed in this form. By a suitable choice of the elements $p_{i}$ in (10) one can make the first $m-1$ factors arbitrary in $S_{2}$ while the last is uniquely determined by the condition (9). The following has been shown therefore:

The group $\bar{R}$ consists of all elements of the form

$$
\rho=\left[r_{1}, \cdots, r_{m-1},\left(r_{1} \cdots r_{m-1}\right)^{-1}\right]
$$

where the $r_{i}$ run through the Abelian group $S_{2}$ independently. The group $\bar{R}$ is isomorphic to the direct product of $m-1$ factors each isomorphic to $S_{2}$.

We can now turn to the final step in the determination of the permutation invariant subgroups $\bar{N}$ of $V_{m}$. Let $\kappa$ in (1) be an arbitrary element of $\bar{N}$ and let

$$
\lambda=\left[k_{2}, k_{1}, k_{3}, \cdots, k_{m}\right]
$$

be the element obtained by interchanging $k_{1}$ and $k_{2}$. Then one finds

$$
\kappa \lambda^{-1}=\left[k_{2}^{-1} k_{1}, k_{1}^{-1} k_{2}, 1, \cdots, 1\right]
$$


showing that the quotient belongs to $\bar{S}_{2}$, and hence $k_{2}{ }^{-1} k_{1}$ is an element of $S_{2}$. Since the same result can be obtained for any $k_{i}^{-1} k_{1}$ one concludes that the elements $\kappa$ of $\bar{N}$ must have the form

$$
\kappa=\left[k r_{1}, k r_{2}, \cdots, k r_{m}\right]
$$

where all the $r_{i}$ are elements of $S_{2}$ and $k$ runs through $K$.

It remains to determine the relations between the $r_{i}$ in (12) which will insure that these elements form a permutation invariant group. Since $\bar{R}$ contains (11) one finds that $\bar{N}$ must contain the element

$$
\rho^{-1} \kappa=\left(k, k, \cdots, k, k^{\prime}\right) .
$$

All elements of this form in $\bar{N}$ must form a group and the element $k^{\prime}$ is uniquely determined by $k$. This correspondence must be a homomorphism and one can write therefore $k^{\prime}=k^{T}$ where $T$ is an endomorphism of $K$. When applied to (11) this gives the final form

$$
\kappa=\left[k r_{1},: \cdots, k r_{m-1}, k^{T}\left(r_{1} \cdots r_{m-1}\right)^{-1}\right]
$$

for the elements belonging to $\bar{N}$.

In (13) one has $k^{\prime}=k r$, where $r$ belongs to $S_{2}$, and hence the endomorphism $T$ of $K$ must transform any element $k$ into an element obtained by multiplication of $k$ by an element in $S_{2}$. When one considers the special element $\left[r, \cdots, r, r^{-(m-1)}\right]$ in $\bar{R}$ it follows that

$$
r^{T}=r^{-(m-1)}
$$

for any element $r$ in $S_{2}$. This relation shows that in (12) the last factor is uniquely determined by the $m-1$ first ones.

Conversely one verifies that under the stated conditions on $T$ and under the assumption that $S_{2}$ is an Abelian group, the elements (14) form a group. Furthermore if two factors in (14) are permuted the quotient between the corresponding substitutions also belongs to the group, and hence it is permutation invariant. We have therefore obtained the principal result:

THEOREM 2. Let $m \geqq 3$. Then all permutation invariant subgroups $\bar{N}$ of $V_{m}(H)$ may be constructed by the following process. A subgroup $K$ of $H$ is chosen. In $K$ two normal subgroups $S_{2} \supset S_{1}$ are selected such that the quotient group $S_{2} / S_{1}$ is Abelian. Then $\bar{N}$ consists of the elements

$$
\kappa=\left[k_{1}, \cdots, k_{m}\right]
$$

where the $k_{i}$ run through $K$ subject to the conditions

$$
\begin{aligned}
k_{i} & \equiv k s_{i}(i=1,2, \cdots, m-1), \\
k_{m} & \equiv k^{T}\left(s_{1} \cdots s_{m-1}\right)^{-1},
\end{aligned}
$$

where the $s_{i}$ are arbitrary elements in $S_{2}$. Furthermore $T$ is an endomorphism of 
$K / S_{1}$ multiplying each element of $K / S_{1}$ by an element in $S_{2} / S_{1}$ and in particular

$$
s^{T} \equiv s^{-(m-1)}\left(\bmod S_{1}\right)
$$

for any element $s$ in $S_{2}$.

Here we have solved the problem of finding all subgroups of $V_{m}(H)$ which are invariant under all permutations of the symmetric group. A much more difficult problem is the determination of those subgroups of $V_{m}(H)$ which are invariant under the permutations of a fixed subgroup of the symmetric group.

4. Normal subgroups of the symmetry contained in the basis group. We shall now turn to the determination of the normal subgroups of the symmetry and we consider first those normal subgroups of $\Sigma_{m}(H)$ which are contained in the basis group. Since we have observed in the preceding that every substitution in $\Sigma_{m}$ is the product of a permutation and a multiplication, we shall have to determine those normal subgroups of $V_{m}$ which are permutation invariant.

We use the same notations as before. If (1) is an element of a normal subgroup $\bar{N}$ of $V_{m}$ the factors $k_{i}$ must run through a normal subgroup $K$ of $H$. Furthermore the subgroups $\bar{S}_{i}$ defined by the elements (5) must also be normal in $V_{m}$ and the corresponding factors $s_{i}$ run through a normal subgroup $S_{i}$ of $H$.

The case $m=2$ must again be considered separately. When $\bar{N}$ contains the element $\kappa=\left[k_{1}, k_{2}\right]$ it must also contain the element $\kappa^{\prime}=\left[h k_{1} h^{-1}, k_{2}\right]$ for an arbitrary $h$ in $H$. But in Theorem 1 the factor $k_{2}$ was uniquely determined by $k_{1}\left(\bmod S_{1}\right)$. This implies that

$$
h k_{1} h^{-1} \equiv k_{1}\left(\bmod S_{1}\right)
$$

for every $h$, and hence $k_{1}$ belongs to the center of $H / S_{1}$. We have therefore:

THEOREM 3. For $m=2$ the normal subgroups of the symmetry which are contained in the basis group can be obtained by the following construction. Two normal subgroups $K \supset S_{1}$ of $H$ are chosen such that $K / S_{1}$ belongs to the center of $H / S_{1}$. Furthermore $T$ is some fixed automorphism of order 2 of $K / S_{1}$. Then the normal subgroups $\bar{N}$ consist of all those elements

$$
\kappa=\left[k_{1}, k_{2}\right]
$$

where $k_{1}$ and $k_{2}$ run through $K$ subject to the condition that

$$
k_{2} \equiv k_{1}^{T}\left(\bmod S_{1}\right) \text {. }
$$

In the general case where $m \geqq 3$ the elements of $\bar{N}$ are given by (14). Here again the last factor is uniquely determined by the $m-1$ first ones. Let us transform $\kappa$ by $\eta=[h, 1, \cdots, 1]$. Then

$$
\eta \kappa \eta^{-1}=\left[h^{-1} k r_{1} h, k r_{2}, \cdots, k r_{m-1}, k^{T}\left(r_{1} \cdots r_{m-1}\right)^{-1}\right] .
$$


This shows first that

$$
h^{-1} k r_{1} h=k s
$$

where $s$ belongs to $S_{2}$, and since the last factor must be the same as before one finds $s=r_{1}$; and hence $k r_{1}$ belongs to the center of $H$.

We have therefore:

THEOREM 4. For $m \geqq 3$ the normal subgroups of $\Sigma_{m}(H)$ contained in the basis group are obtained by the construction in Theorem 2 with the additional condition that $K \supset S_{2} \supset S_{1}$ are normal subgroups of $H$ such that $K / S_{1}$ belongs to the center of $H / S_{1}$.

5. Other normal subgroups of $\Sigma_{m}(H)$. We shall complete our investigations of the normal subgroups of the symmetry by the determination of those normal subgroups which are not contained in the basis group.

Let $\bar{M}$ be such a normal subgroup of $\Sigma_{m}$. The cross-cut

$$
\bar{N}=\bar{M} \cap V_{m}
$$

consists of the multiplications contained in $\bar{M}$ and $\bar{N}$ is normal in $\Sigma_{m}$. Since $\bar{N}$ is contained in $V_{m}$ it must have one of the forms determined in the preceding. But in this case one can make a further statement about $\bar{N}$.

Let $\alpha$ be a substitution in $\bar{M}$ and

$$
\gamma=\left(\begin{array}{ll}
x_{1}, & x_{2}, \cdots, x_{n} \\
c_{1} x_{2}, & c_{2} x_{3}, \cdots, c_{n} x_{1}
\end{array}\right)
$$

a cycle in $\alpha$, while

$$
\eta=\left[h_{1}, \cdots, h_{m}\right]
$$

is an arbitrary multiplication in $V_{m}$. Since $\bar{M}$ is normal in $\Sigma_{m}$ it must contain the multiplication defined by the commutator

$$
\eta \alpha \eta^{-1} \alpha^{-1}=\left[\cdots, c_{n}^{-1} h_{n}^{-1} c_{n} h_{1}, c_{1}^{-1} h_{1}^{-1} c_{1} h_{2}, \cdots\right]
$$

where only the factors corresponding to the cycle $\boldsymbol{\gamma}$ have been indicated. Since the elements $h_{i}$ in $H$ are arbitrary it follows that the factor at any position can be made to take an arbitrary value. Using these results we can say:

TheOREM 5. Let $\bar{M}$ be a normal subgroup of $\Sigma_{m}$ not contained in the basis group. The multiplications contained in $\bar{M}$ form a normal subgroup $\bar{N}$ of $\Sigma_{m}$ in which $H=K$, i.e., the factors in any fixed position run through the whole group and the quotient $H / S_{1}$ for $\bar{N}$ is an Abelian group.

Again we shall have to consider the case $m=2$ separately. In this case every element which is not a multiplication is a cycle of length 2 and every element in $\bar{M}$ must belong to one of the cosets 


$$
\bar{M}=\bar{N}+\tau \bar{N}
$$

where $\tau$ is any fixed cycle,

$$
\tau=\left(\begin{array}{ll}
x_{1}, & x_{2} \\
x_{2}, & a x_{1}
\end{array}\right)
$$

in $\bar{M}$. Since

$$
\tau^{2}=[a, a]
$$

must belong to $\bar{N}$ it follows from Theorem 3 that

$$
a^{T} \equiv a\left(\bmod S_{1}\right),
$$

i.e., $a$ is an element in $H$ invariant under the automorphism $T$ of $H / S_{1}$.

Now let us consider a group $\bar{M}$ defined by (15) where $\tau$ is the cycle (16) and $\bar{N}$ is a normal subgroup of $\Sigma_{2}$ contained in $V_{2}$ having the properties indicated in Theorem 5. If the condition (17) is satisfied it is obvious that the elements defined by (15) form a group. In order that it should be normal in $\Sigma_{2}$ it is necessary and sufficient that it be transformed into itself by any multiplication

$$
\kappa=\left[h_{1}, h_{2}\right]
$$

and by the transposition $\sigma=(1,2)$. Since $\bar{N}$ already is a normal subgroup of $\Sigma_{m}$ it is sufficient to show that $\tau$ is transformed into an element of $\bar{M}$. This in turn is equivalent to the fact that the commutators

$$
\kappa \tau \kappa^{-1} \tau^{-1}, \quad \sigma \tau \sigma^{-1} \tau^{-1}
$$

shall belong to $\bar{N}$.

For the first commutator one finds

$$
\kappa \tau \kappa^{-1} \tau^{-1}=\left[a^{-1} h_{2}^{-1} a h_{1}, h_{1}^{-1} h_{2}\right] .
$$

This implies that

$$
a^{-1} h_{2}^{-1} a h_{1} \equiv\left(h_{1}^{-1} h_{2}\right)^{T}\left(\bmod S_{1}\right)
$$

and since $H / S_{1}$ is Abelian

$$
\left(h_{1}^{-1} h_{2}\right)^{T} \equiv\left(h_{1}^{-1} h_{2}\right)^{-1}\left(\bmod S_{1}\right),
$$

hence $T$ is an automorphism of $H / S_{1}$ taking each element into its inverse.

For the second commutator one finds

$$
\sigma \tau \sigma^{-1} \tau^{-1}=\left[a^{-1}, a\right]
$$

which is always in $\bar{N}$. By the condition (17) one must also have $a^{2}=1$.

THEOREM 6. The normal subgroups of $\Sigma_{2}(H)$ not contained in $V_{2}(H)$ can 
be obtained by the following construction. A normal subgroup $S_{1}$ of $H$ is chosen so that $H / S_{1}$ is Abelian. Then the group $\bar{N}$ consisting of the elements

$$
\kappa=\left[k_{1}, k_{2}\right]
$$

where

$$
k_{2} \equiv k_{1}^{-1}\left(\bmod S_{1}\right)
$$

forms a normal subgroup of $\Sigma_{2}$ contained in $V_{2}$. The general normal subgroup $\bar{M}$ of $\Sigma_{2}$ not in $V_{2}$ consists of the two cosets

$$
\bar{M}=\bar{N}+\tau \bar{N}
$$

where

$$
\tau=\left(\begin{array}{l}
x_{1}, x_{2} \\
x_{2}, a x_{1}
\end{array}\right)
$$

with

$$
a^{2} \equiv 1\left(\bmod S_{1}\right)
$$

We shall now turn to the general case where $m \geqq 3$. As before we denote by $\bar{M}$ a normal subgroup of $\Sigma_{m}$ not contained in $V_{m}$ and by $\bar{N}$ the cross-cut of $\bar{M}$ with $V_{m}$. Furthermore we denote by $P$ the subgroup of $\bar{M}$ consisting of permutations only. Since $\bar{M}$ is normal it follows that $P$ is normal in the symmetric group $S_{m}$.

We shall prove first:

TheOREM 7. Every normal subgroup $\bar{M}$ of $\Sigma_{m}, m \geqq 3$, not contained in $V_{m}$ contains permutations.

Proof. Let $\alpha$ be a substitution in $\bar{M}$ and $\gamma$ a cycle in $\alpha$ of length $n \geqq 2$. According to a previous result $\alpha$ can always be so transformed that

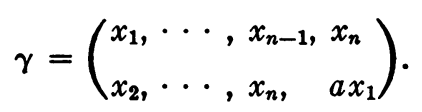

We shall now have to separate several cases. Let us assume first $n \geqq 3$. Then one puts $\tau=(1,2)$ and one finds that

$$
\tau \alpha \tau^{-1} \alpha^{-1}=(1,2,3)
$$

belongs to $P$.

We can assume therefore that every cycle which occurs in any substitution in $\bar{M}$ has the lengths 1 and 2. If $\alpha$ contains at least two cycles of length 2 one can write

$$
\alpha=\left(\begin{array}{l}
x_{1}, x_{2} \\
x_{2}, a x_{1}
\end{array}\right)\left(\begin{array}{l}
x_{3}, x_{4} \\
x_{4}, b x_{3}
\end{array}\right) \cdots
$$


If one puts $\tau=(1,3)$ one obtains

$$
\tau \alpha \tau^{-1} \alpha^{-1}=(1,3)(2,4) .
$$

Finally if $\alpha$ contains only one cycle of length 2 one can write

$$
\alpha=\left(\begin{array}{ll}
x_{1}, x_{2}, & x_{3}, \cdots \\
x_{2}, a x_{1}, b x_{3}, \cdots
\end{array}\right) \text {. }
$$

When $\tau=(1,3)$ one finds

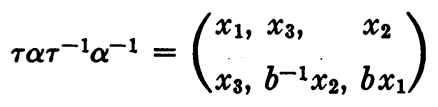

and $\bar{M}$ contains a cycle of length 3 contrary to assumption.

Since $P$ is a normal subgroup of the symmetric group, there exist only the following three possibilities:

1. $P$ is the symmetric group $S_{m}$.

2. $P$ is the alternating group $A_{m}$.

3. When $m=4, P$ can be the four group $P_{4}$ consisting of the elements

$$
1,(1,2)(3,4),(1,3)(2,4),(1,4)(2,3) \text {. }
$$

On the basis of this result one can prove a stronger statement than Theorem 5:

THEOREM 8. Let $\bar{M}$ be a normal subgroup of $\Sigma_{m}(H), m \geqq 3$, not contained in the basis group. Then the multiplications in $\bar{M}$ form a normal subgroup $\bar{N}$ consisting of the elements

$$
\eta=\left[h_{1}, \cdots, h_{m}\right]
$$

where the $h_{i}$ run through $H$ subject to the condition

$$
h_{1} \cdots h_{m} \equiv 1\left(\bmod S_{1}\right) \text {. }
$$

Here $S_{1}$ is a normal subgroup of $H$ such that $H / S_{1}$ is Abelian.

Proof. Let us assume first that the group of permutations $P$ in $\bar{M}$ is the symmetric or alternating group. Then it contains the cycle $\tau=(1,2,3)$, and hence also the commutator

$$
\kappa \tau \kappa^{-1} \tau^{-1}=\left[k_{3}^{-1} k_{1}, k_{1}^{-1} k_{2}, k_{2}^{-1} k_{3}, 1, \cdots, 1\right]
$$

where

$$
\kappa=\left[k_{1}, \cdots, k_{m}\right]
$$

is an arbitrary multiplication. But in (20) two factors may be made perfectly arbitrary. Hence when one compares it with the group in Theorem 2 one must have $S_{2}=H$ and an element (18) in $\bar{N}$ is subject only to condition (19). 
In the exceptional case when $P=P_{4}$ is the four group, it must contain $(1,2)(3,4)=\tau$ and

$$
\kappa \tau \kappa^{-1} \tau^{-1}=\left[k_{2}^{-1} k_{1}, k_{1}^{-1} k_{2}, k_{4}^{-1} k_{3}, k_{3}^{-1} k_{4}, 1, \cdots, 1\right]
$$

belongs to $\bar{N}$. Here the third and the first factors may take arbitrary values in $H$ and the same argument as before applies.

We may also observe that in Theorem 8 the quotient group $V_{m} / \bar{N}$ is Abelian and this holds even when $m=2$.

We shall now proceed to the actual construction of the normal subgroups of $\Sigma_{m}$. The quotient group $\Sigma_{m} / V_{m}$ is isomorphic to the symmetric group. Furthermore

$$
\bar{M} \cup V_{m} / V_{m} \simeq \bar{M} / \bar{N}
$$

is a group isomorphic to a normal subgroup of $S_{m}$, and hence the group $\bar{M} / \bar{N}$ is isomorphic to either $S_{m}, A_{m}$ or $P_{4}$.

The group $P$ was also isomorphic to one of these groups. Let us now suppose first that $P \simeq \bar{M} / \bar{N}$ and hence $\bar{M}=P \cup \bar{N}$. We then have the following theorem:

THEOREM 9. Let $P$ be a normal subgroup of the symmetric group and $\bar{N} a$ normal subgroup of the basis group of the type defined in Theorem 8. Then

$$
\bar{M}=P \cup \bar{N}
$$

is a normal subgroup of $\Sigma_{m}$.

Proof. The group $\bar{N}$ is normal in $\Sigma_{m}$ by assumption, and hence it is only necessary to show that an element in $P$ is transformed into an element in $\bar{M}$. Since $P$ is normal in $S_{m}$ it is sufficient to show that a permutation $\pi$ in $P$ is transformed into an element in $\bar{M}$ by any multiplication

$$
\kappa=\left[k_{1}, \cdots, k_{m}\right] .
$$

But if one forms the commutator

$$
\kappa \pi \kappa^{-1} \pi^{-1}=\left[\cdots, h_{\pi^{-1}: i k_{i}}^{-1}, \cdots\right]
$$

one sees, since $H / S_{1}$ is Abelian, that the condition (19) is satisfied.

It remains therefore only to consider the case where $P$ is isomorphic to a proper subgroup of $\bar{M} / \bar{N}$. This can only happen in the following two cases:

1. $P=P_{4}$ and $\bar{M} / \bar{N} \simeq S_{m}$ or $\bar{M} / \bar{N} \simeq A_{m}$.

2. $P=A_{m}$ and $\bar{M} / \bar{N} \simeq S_{m}$.

The first case is easily disposed of. If $\bar{M} / \bar{N}$ is isomorphic to $S_{m}$ or $A_{m}$ it follows that $\bar{M}$ must contain a cycle of length 3 and by the argument used in the proof of Theorem 7 it follows that $P$ contains a cycle $(1,2,3)$ contrary to the assumption that $P=P_{4}$. 
We consider therefore the second case where $P=A_{m}$. Then there must exist some permutation

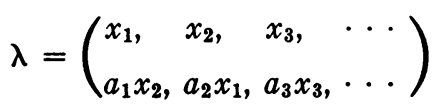

in $\bar{M}$. According to Theorem 8 the factors in any element in $\bar{N}$ may be taken arbitrarily in $H$. except for one of them and it is therefore no limitation to assume $a_{3}=\cdots=a_{m}=1$. Furthermore one sees that $\lambda$ may be so transformed that

$$
\lambda=\left(\begin{array}{c}
x_{1}, x_{2} \\
x_{2}, a x_{1}
\end{array}\right) \text {. }
$$

Since $\lambda^{2}$ must belong to $\bar{N}$ one finds

$$
a^{2} \equiv 1\left(\bmod S_{1}\right) \text {. }
$$

We can then prove:

THEOREM 10. Let

$$
\bar{M}_{A}=A_{m} \cup \bar{N}
$$

be a normal subgroup of $\Sigma_{m}$ defined by the procedure of Theorem 9 and let $L=\{\lambda\}$ be the cyclic subgroup defined by a cycle (21), where a satisfies the condition (22). Then

$$
\bar{M}=L \cup \bar{M}_{A}
$$

is a normal subgroup of $\Sigma_{m}$.

Proof. As before it is sufficient to prove that the commutator of $\lambda$ with a permutation and with a multiplication again belongs to $\bar{M}$ and this is easily verified.

We can now summarize the preceding investigation as follows:

For $m=2$ the normal subgroups of the symmetry are of the forms given in Theorem 3 and Theorem 6.

For $m \geqq 3$ the normal subgroups of the symmetry are of the forms determined by Theorem 4, Theorem 9 and Theorem 10 .

6. Commutator groups. We shall now determine the commutator group $\Sigma_{m}^{\prime}$ of the symmetry $\Sigma_{m}(H)$. The commutator group of $H$ shall be denoted by $H^{\prime}$. It is then easily seen that the commutator group $V_{m}^{\prime}$ of the basis group consists of all elements

$$
\eta^{\prime}=\left[h_{1}^{\prime}, \cdots, h_{m}^{\prime}\right]
$$

where the $h_{i}^{\prime}$ run through the elements of $H^{\prime}$ independently.

From the well known properties of the symmetric group it follows that 
the commutator group of the symmetric group is equal to the alternating group $A_{m}$ for all $m$, when one puts $A_{2}=1$ in the special case $m=2$. Thus the commutator group $\Sigma^{\prime}$ must contain $A_{m}$.

Next let us form the commutator of the two elements $\left(x_{1}, x_{2}\right)$ and $[h, 1, \cdots, 1]$ where $h$ is an arbitrary element in $H$. One finds that this commutator is the multiplication

$$
\left[h^{-1}, h, 1, \cdots, 1\right] .
$$

From this result one concludes as in $\S 3$ that $\Sigma^{\prime}$ also contains the group $V_{m}^{*}(H)$ consisting of all multiplications

$$
\eta=\left[h_{1}, \cdots, h_{m}\right]
$$

where

$$
h_{1} h_{2} \cdots h_{m} \equiv 1\left(\bmod H^{\prime}\right) .
$$

The quotient group $V / V^{*}$ is seen to be Abelian and isomorphic to the group $H / H^{*}$.

From these remarks one can conclude:

THEOREM 11. The commutator group of the symmetry is

$$
\Sigma_{m}^{\prime}(H)=A_{m} \cup V_{m}^{*}
$$

where $A_{m}$ is the alternating group and $V_{m}^{*}$ the normal subgroup of $\Sigma_{m}$ contained in $V_{m}$ consisting of the elements (23) which satisfy the condition (24).

Proof. It has already been shown that the commutator group must contain $A_{m}$ and $V_{m}{ }^{*}$. Thus it is sufficient to show that the quotient group $\Sigma_{m} / A_{m} \cup V_{m}^{*}$ is Abelian. Obviously any two multiplications commute $\left(\bmod V_{m}^{*}\right)$ since this group contains $V_{m}^{\prime}$. Furthermore the quotient group is seen to be generated by residue classes defined by multiplications and the transposition $\left(x_{1}, x_{2}\right)$ and these generating classes are also seen to commute $\left(\bmod A_{m} \cup V_{m}^{*}\right)$.

Next let us determine the second commutator group $\Sigma_{m}^{\prime \prime}(H)$. Since the alternating group is simple, one has $A_{m}{ }^{\prime}=A_{m}$ except for the well known exceptions,

$$
A_{2}^{\prime}=1, \quad A_{3}^{\prime}=1, \quad A_{4}^{\prime}=P_{4} .
$$

These remarks show that for $m \geqq 5$ the group $\Sigma_{m}^{\prime \prime}$ must contain $A_{m}$ except for $m=4$ when it contains $P_{4}$.

We shall now determine certain multiplications which must be contained in $\Sigma^{\prime \prime}$. Let us suppose that $m \geqq 3$.

If $h_{1}$ and $h_{2}$ are arbitrary elements in $H$ then $\Sigma^{\prime}$ contains the multiplications

$$
\eta_{1}=\left[h_{1}, h_{1}^{-1}, 1, \cdots, 1\right], \quad \eta_{2}=\left[h_{2}, 1, h_{2}^{-1}, 1, \cdots, 1\right]
$$


By forming the commutator it follows that $\Sigma^{\prime \prime}$ contains

$$
\eta_{1} \eta_{2} \eta_{1}^{-1} \eta_{2}^{-1}=\left[h_{1} h_{2} h_{1}^{-1} h_{2}^{-1}, 1, \cdots, 1\right]
$$

hence $\Sigma^{\prime \prime}$ must contain $V_{m}^{\prime}$.

We shall now prove that in most cases $\Sigma^{\prime \prime}$ contains the group $V_{m}^{*}$. To obtain this result let $n \geqq 3$ be some odd number not exceeding $m$. The cycle

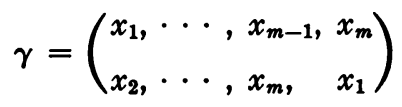

then belongs to $A_{m}$. Furthermore let

$$
\eta=\left[h_{1}, \cdots, h_{n}, 1, \cdots, 1\right]
$$

with

$$
h_{1} h_{2} \cdots h_{n} \equiv 1\left(\bmod H^{\prime}\right)
$$

be an element in $V_{m}{ }^{*}$. The commutator

$$
\pi=\eta^{-1} P^{-1} \eta P=\left[h_{2} h_{1}^{-1}, h_{3} h_{2}^{-1}, \cdots, h_{n} h_{n-1}^{-1}, h_{1} h_{n}^{-1}, 1, \cdots, 1\right]
$$

must then belong to $\Sigma^{\prime \prime}$. We shall consider $\pi\left(\bmod V_{m}^{\prime}\right)$ and eliminate $h_{n}$ by means of the condition (25). Since $H / H^{\prime}$ is Abelian one finds

$$
\begin{aligned}
& \pi \equiv\left[h_{2} h_{1}^{-1}, h_{3} h_{2}^{-1}, \cdots, h_{1}^{-1} \cdots h_{n-2}^{-1} h_{n-1}^{-2}, h_{1}^{2} h_{2} \cdots h_{n-1}, 1, \cdots, 1\right] \\
& \left(\bmod V_{m}^{\prime}\right)
\end{aligned}
$$

and here the $h_{i}(i=1, \cdots, n-1)$ can take on arbitrary values in $H$.

For $h_{1}=1$ the first multiplier in (26) becomes the arbitrary element $h_{2}$. Thus the group of multiplications in $\Sigma^{\prime \prime}$ must have the property that the multiplier at any position runs through $H$.

Next let us make all $h_{i}(i=1,2, \cdots, n-1)$ equal to the same element $h$. From (26) one obtains

$$
\pi \equiv\left[1, \cdots, 1, h^{-n}, h^{n}, 1, \cdots, 1\right]\left(\bmod V_{m}^{\prime}\right)
$$

and one sees that $\Sigma^{\prime \prime}$ contains

$$
\eta_{n}=\left[h^{-n}, h^{n}, 1, \cdots, 1\right]
$$

for any odd $n$ such that $m \geqq n \geqq 3$.

If now $m \geqq 5$ one can take $n=3$ and $n=5$ and by combining the results one finds that $\Sigma^{\prime \prime}$ contains

$$
\eta_{1}=\left[h^{-1}, h, 1, \cdots, 1\right],
$$

hence it contains the full group $V_{m}^{*}$. 
In the special case $m=4$ one can obtain the same result as follows. The alternating group $A_{4}$ contains the substitution

$$
\gamma_{1}=\left(\begin{array}{l}
x_{1}, x_{2} \\
x_{2}, x_{1}
\end{array}\right)\left(\begin{array}{l}
x_{3}, x_{4} \\
x_{4}, x_{3}
\end{array}\right)
$$

and one finds by the same process of forming commutators that $\Sigma_{4}^{\prime \prime}$ must contain $\eta_{4}$ and since it contains $\eta_{3}$ it also contains $\eta_{1}$. Thus it has been shown:

TheOREM 12. For $m \geqq 5$ the second commutator group $\Sigma_{m}^{\prime \prime}(H)$ is equal to the first commutator group $\Sigma_{m}^{\prime}(H)$. For $m=4$ one has

$$
\Sigma_{4}^{\prime \prime}(H)=P_{4} \cup V_{4}^{*}
$$

where $V_{4}^{*}$ consists of all multiplications

$$
\eta=\left[h_{1}, h_{2}, h_{3}, h_{4}\right]
$$

with

$$
h_{1} h_{2} h_{3} h_{4} \equiv 1\left(\bmod H^{\prime}\right) .
$$

In the case $m=3$ the second commutator group is contained in the basis group, hence it must be a normal subgroup of the form determined by Theorem 9. We mention the result without proof:

THEOREM 13. For $m=3$ the second commutator group $\Sigma_{3}^{\prime \prime}(H)$ is a subgroup of the basis group consisting of the multiplications $\eta=\left[h_{1}, h_{2}, h_{3}\right]$ where the factors are subject to the conditions

$$
h_{1} \equiv h k_{1}^{3}, h_{2} \equiv h k_{2}^{3}, h_{3} \equiv h^{-2}\left(k_{1} k_{2}\right)^{-3}\left(\bmod H^{\prime}\right)
$$

and $h, k_{1}, k_{2}$ are arbitrary elements in $H$.

One finally finds:

TheOREM 14. For $m=2$ the second commutator group $\Sigma_{2}^{\prime}(H)$ consists of the multiplications

$$
\eta=\left[h_{1}, h_{2}\right]
$$

where $h_{1}$ and $h_{2}$ run through $H^{\prime}$ subject to the condition

$$
h_{2} \equiv h_{1}(\bmod \bar{H})
$$

where $\bar{H}$ is the group generated by the commutators of an element in $H$ with an element in $H^{\prime}$.

For $m=2,3,4$ one can also determine the higher commutator groups. One can also, through similar methods, determine the commutator groups of the normal subgroups of the symmetry. 


\section{Chapter III. Automorphisms}

1. The basis group as characteristic subgroup. We shall now turn to the problem of the determination of the automorphisms of the symmetry. Again the case $m=2$ presents a real exception and we shall assume, therefore, to begin with that $m \geqq 3$. We shall assume also that the group $H$ with respect to which the symmetry $\Sigma_{m}(H)$ is constructed is a finite group. This condition is, however, not essential in most of the results, but it simplifies several of the special considerations which are necessary for the lowest values of $m$.

We begin our investigations by deducing the following theorem:

THEOREM 1. For $m \geqq 3$ the basis group is a characteristic subgroup of the symmetry.

Proof. Let us assume that the theorem is not true. Then there would exist some automorphism $\alpha$ which takes a subgroup $\bar{M}$ into the basis group $V_{m}$. The group $\bar{M}$ is a normal subgroup of $\Sigma_{m}$ not contained in the basis group and hence its form is determined by Theorem 6, Chapter II.

The quotient group $\Sigma_{m} / V_{m}$ is isomorphic to the symmetric group $S_{m}$. Since $\bar{M}$ is transformed into $V_{m}$ by $\alpha$ one must also have

$$
\Sigma_{m} / \bar{M} \simeq S_{m} \simeq \Sigma_{m} / V_{m}
$$

Next let us write

$$
\bar{K}=\bar{M} \cup V_{m}, \quad \bar{N}=\bar{M} \cap V_{m} .
$$

Since $\bar{K}$ is a normal subgroup of $\Sigma_{m}$ containing $V_{m}$ there exist only the following three possibilities

$$
\bar{K}=S_{m} \cup V_{m}, \quad \bar{K}=A_{m} \cup V_{m}, \quad \bar{K}=P_{4} \cup V_{4},
$$

where as before $A_{m}$ denotes the alternating group and $P_{4}$ the four group.

According to (1) the two quotient groups $\Sigma_{m} / V_{m}$ and $\Sigma_{m} / \bar{M}$ are isomorphic. Thus the quotient groups $\bar{K} / V_{m}$ and $\bar{K} / \bar{M}$ must have the same order and since both of them are isomorphic to a normal subgroup of $S_{m}$ one must have

$$
\bar{K} / \bar{M} \simeq \bar{K} / V_{m} .
$$

Then one concludes from the second law of isomorphism

$$
\bar{K} / V_{m} \simeq \bar{M} / \bar{N} \simeq V_{m} / \bar{N}
$$

The form of the normal subgroup $\bar{N}$ can also be determined from Theorem 6, Chapter II. According to this theorem there must exist a normal subgroup $S$ of $H$ such that $H / S$ is Abelian. Then $\bar{N}$ consists of all multiplications

$$
\eta=\left[h_{1}, \cdots, h_{m}\right]
$$


where the elements $h_{i}$ run through $H$ subject only to the condition

$$
h_{1} \cdots h_{m} \equiv 1(\bmod S) \text {. }
$$

This shows that the quotient group $V_{m} / \bar{N}$ is isomorphic to $H / S$, hence $S$ is a proper subgroup of $H$. But furthermore, since $V_{m} / \bar{N}$ is Abelian it follows from (2) and (3) that one is led to a contradiction in all cases except possibly in the two special cases

$$
\begin{array}{ll}
m=3, & \bar{M} / \bar{N} \simeq A_{3}, \\
m=4, & \bar{M} / \bar{N} \simeq P_{4} .
\end{array}
$$

To prove the theorem also for these two cases we proceed somewhat differently. Since the two groups $\bar{M}$ and $V_{m}$ are isomorphic, their commutator groups $\bar{M}^{\prime}$ and $V_{m}^{\prime}$ and also the corresponding quotient groups $\bar{M} / \bar{M}^{\prime}$ and $V_{m} / V_{m}^{\prime}$ must be isomorphic.

The group $V_{m}^{\prime}$ is seen to consist of the elements

$$
\kappa=\left[k_{1}, \cdots, k_{m}\right]
$$

where the $k_{i}$ run through the commutator group $H^{\prime}$ of $H$. We can now show that in the case $m \geqq 3$ the commutator group of the cross-cut $\bar{N}$ of $\bar{M}$ and $V_{m}$ consists of the same elements (4). When $h_{1}$ and $h_{2}$ are arbitrary elements in $H$ the group $\bar{N}$ must contain the two elements

$$
\left[h_{1}, h_{1}^{-1}, 1, \cdots, 1\right], \quad\left[h_{2}, 1, h_{2}^{-1}, 1, \cdots, 1\right] ;
$$

thus the commutator group $\bar{N}^{\prime}$ must contain

$$
\left[h_{1} h_{2} h_{1}^{-1} h_{2}^{-1}, 1, \cdots, 1\right]
$$

and consequently $\bar{N}^{\prime}=V_{m}^{\prime}$.

We obtain the desired contradiction by showing that $\bar{M}^{\prime}$ contains $V_{m}^{\prime}$ as a proper subgroup. According to Theorem 7, Chapter II, there exists some permutation in $\bar{M}$ and the proof of this theorem shows that there must exist some permutation $P$ in $\bar{M}$ taking $x_{1}$ into $x_{2}$. But then for any element

the commutator

$$
\eta=\left[h_{1}, \cdots, h_{m}\right]
$$

$$
\eta^{-1} P^{-1}{ }^{-1} P=\left[h_{2} h_{1}^{-1}, \cdots\right]
$$

must belong to $\bar{M}^{\prime}$. By a suitable choice of $h_{1}$ and $h_{2}$ one obtains that in $\bar{M}^{\prime}$ the factors of any variable in a multiplication can run through the full group $H$. But according to the construction of $\bar{N}$ the group

$$
S \supset H^{\prime}
$$

is a proper subgroup of $H$ hence $\bar{M}^{\prime}$ contains $V_{m}^{\prime}$ as a proper subgroup.

The result obtained in Theorem 1 can be supplemented as follows: 
THEOREM 2. In the case $m=2$ the basis group need not be a characteristic subgroup of the symmetry.

To prove this statement we turn to the simplest example of a symmetry where $H$ is a cyclic group of order 2 generated by an element $a$ for which $a^{2}=1$. Then the group $\Sigma_{2}(H)$ consists of the following eight eiements:

$$
\begin{gathered}
1,[a, a],[a, 1],[1, a], \\
\left(\dot{x}_{1}, x_{2}\right),\left(x_{1}, x_{2}\right)[a, a],\left(x_{1}, x_{2}\right)[a, 1],\left(x_{1}, x_{2}\right)[1, a] .
\end{gathered}
$$

If one puts $x=\left(x_{1}, x_{2}\right), y=\left(x_{1}, x_{2}\right)[a, 1]$, then the group $\Sigma_{2}(2)$ can also be defined by the generating relations

$$
x^{2}=1, \quad y^{4}=1, \quad x y x^{-1}=y^{3} .
$$

We shall now determine the group of outer automorphisms of this group. The two first elements in (5) constitute the center of the group and they are, therefore, invariant by all automorphisms. Since the two last elements in (5) are the only ones of order 4 , any automorphism $\alpha$ must take $y$ into $y$ or $y^{3}$. But in the latter case one can, according to (6), multiply $\alpha$ by the inner automorphism $x$ and one can, therefore, always assume

$$
y^{\alpha}=y \text {. }
$$

The element $x$ must be transformed under $\alpha$ into some other element of order 2 . It cannot be left invariant because then according to ( 7$)$ every element would be invariant. It cannot be transformed into $[a, a]$ since this element belongs to the center. There remain, therefore, only the three possibilities

$$
x^{\alpha}=[a, 1], \quad x^{\alpha}=[1, a], \quad x^{\alpha}=\left(x_{1}, x_{2}\right)[a, a] .
$$

The last alternative in (8) can be ruled out since one finds that it corresponds to the inner automorphism defined by $y$. One finds that the two remaining correspondences (8) differ only by an inner automorphism and that they actually define an outer automorphism

$$
\begin{aligned}
\left(x_{1}, x_{2}\right)^{\alpha} & =[a, 1], & & {[a, 1]^{\alpha}=\left(x_{1}, x_{2}\right)[a, a], } \\
\left(\left(x_{1}, x_{2}\right)[a, a]\right)^{\alpha} & =[1, a], & & {[1, a]^{\alpha}=\left(x_{1}, x_{2}\right) . }
\end{aligned}
$$

Since this automorphism does not leave the basis group invariant, Theorem 2 has been proved.

2. The automorphisms of the basis group. Before we proceed to the determination of the automorphisms of the symmetry it is necessary to make some remarks about the automorphisms of the basis group. This is of course the same as the determination of the automorphisms of a direct product of $m$ factors all isomorphic to $H$.

Let $H$ be some group. An endomorphism of $H$ is a homomorphic image of $H$ upon some subgroup. Since any homomorphic image is isomorphic to 
some quotient group $H / A$ all endomorphisms are obtainable from the subgroups of $H$ isomorphic to some such quotient group.

The endomorphisms of the basis group $V_{m}(H)$ can easily be determined from those of the group $H$. The group $V_{m}(H)$ is the direct product of the $m$ isomorphic groups $\bar{H}^{(i)}$ each consisting of the elements

$$
\eta_{i}=\left[1, \cdots, 1, h_{i}, 1, \cdots, 1\right] .
$$

Now let $T$ be some endomorphism of $V_{m}(H)$. Then one must have

$$
\eta_{i}^{T}=\left[h_{i}^{\boldsymbol{T}_{i}^{(i)}}, \cdots, h_{i}^{T^{(i)}}\right]
$$

where the $T_{s}^{(i)}$ denote correspondences in $H$. One sees immediately that these correspondences must be endomorphisms in $H$. Furthermore, since the two elements $\eta_{i}$ and $\eta_{j}$ are permutable one finds that

$$
h^{T_{s}^{(i)}} k^{T_{s}^{(i)}}=k^{T_{s}^{(j)}} h^{T_{s}^{(i)}} \quad(s=1,2, \cdots, m), i \neq j,
$$

for arbitrary elements $h$ and $k$ in $H$.

Through the correspondences (11) one finds the element corresponding to the general element

$$
\eta=\left[h_{1}, \cdots, h_{m}\right]
$$

to be

$$
\eta^{T}=\left[h_{1}^{T_{1}^{(1)}} h_{2}^{T_{1}^{(2)}} \cdots h_{m}^{T_{1}^{(m)}}, h_{1}^{T_{2}^{(1)}} h_{2}^{T_{2}^{(2)}} \cdots h_{m}^{T_{2}^{(m)}}, \cdots\right] .
$$

Conversely one sees that if a set of endomorphisms

$$
T_{s}^{(i)} \quad(s=1,2, \cdots, m ; i=1,2, \cdots, m)
$$

are given and the element $\eta^{T}$ corresponding to $\eta$ in (13) is given by (14) then $T$ represents an endomorphism of $V_{m}(H)$ if the conditions (12) are satisfied.

THEOREM 3. All endomorphisms of $V_{m}(H)$ are obtainable through the possible sets of $m^{2}$ endomorphisms (15) of $H$ satisfying (12), by letting the general element $\eta$ in (13) correspond to the element (14).

The endomorphism thus defined is an automorphism if and only if the set of equations

$$
h_{1}^{T_{i}^{(1)}} h_{2}^{T_{i}^{(2)}} \cdots h_{m}^{T_{i}^{(m)}}=k_{i} \quad(i=1,2, \cdots, m)
$$

have a unique solution $h_{1}, h_{2}, \cdots, h_{m}$ for arbitrary $k_{1}, k_{2}, \cdots, k_{m}$ in $H$.

3. Conditions on automorphisms. We shall now proceed to the actual determination of the outer automorphisms of the symmetry in the general case. 
These investigations are based upon the following remarks. Let $G$ be a group and $N$ some normal subgroup of $G$ such that $G$ splits over $N$

$$
G=M \cup N ; \quad M \cap N=E .
$$

Now let $M^{\prime}$. and $N^{\prime}$ be groups isomorphic to $M$ and $N$ under the isomorphisms $\alpha$ and $\beta$, respectively. Then to each element $\mu \cdot \nu$ in $G$ can be made to correspond a unique symbol $\mu^{\alpha} \cdot \nu^{\beta}$. Now let us determine when this correspondence defines an isomorphism between $G$ and a group

$$
G^{\prime}=M^{\prime} \cup N^{\prime}, \quad M^{\prime} \cap N^{\prime}=E .
$$

Since the multiplication in $G$ is defined by the rule

one must have

$$
\mu_{1} \nu_{1} \cdot \mu_{2} \nu_{2}=\mu_{1} \mu_{2} \cdot \mu_{2}^{-1} \nu_{1} \mu_{2} \cdot \nu_{2}
$$

$$
\underset{\mu_{1} \nu_{1} \cdot \mu_{2} \nu_{2}}{\alpha \beta}=\left(\mu_{1} \mu_{2}\right)^{\alpha} \cdot\left(\mu_{2}^{-1} \nu_{1} \mu_{2}\right)^{\beta} \cdot \nu_{2}^{\beta}
$$

This reduces to the necessary and sufficient condition

$$
\mu^{\alpha} \nu^{\beta}\left(\mu^{-1}\right)^{\alpha}=\left(\mu \nu \mu^{-1}\right)^{\beta}
$$

which must hold for all $\mu$ in $M$ and $\nu$ in $N$.

For the symmetry one can always write according to $\$ 1$, Chapter I,

$$
\Sigma_{m}=S_{m} \cup V_{m}, \quad S_{m} \cap V_{m}=E
$$

where $S_{m}$ is the symmetric group and $V_{m}$ the basis group. This relation corresponds to the preceding (16). Now let $\tau$ be any automorphism of $\Sigma_{m}$. Since $V_{m}$ is a characteristic subgroup for $m \geqq 3$ according to Theorem 1, one obtains from (18)

$$
\Sigma_{m}=S_{m}^{\tau} \cup V_{m}, \quad S_{m}^{\tau} \cap V_{m}=E .
$$

The possible form of such a group $S_{m}^{\tau}$ is determined by Theorem 10, Chapter I. There exists an isomorphism $\tau$ between $S_{m}$ and $S_{m}^{\tau}$ generated by the correspondence

$$
(1, i)^{r}=\left[1, a_{1, i}, \cdots, a_{m, i}\right](1, i)
$$

which indicates the elements corresponding to the transpositions $(1, i)$ generating $S_{m}$. Here the $a_{i, j}$ are elements of $H$ generating a subgroup of $H$ homomorphic to the symmetric group on $m-1$ letters by means of the correspondence

$$
(i, j) \rightarrow a_{i, j}, \quad i \neq 1, j \neq 1 .
$$

We have now the same situation as in the introductory remarks. There exists an isomorphism $\alpha$ between $S_{m}$ and $S_{m}^{r}$, hence $\tau$ and $\alpha$ can only differ in their effect upon $S_{m}$ by an automorphism of $S$. But according to a result due 
to $H$ ölder $\left({ }^{3}\right)$, the symmetric group on $m$ letters has only inner automorphisms except when $m=6$. Since we are only interested in outer automorphisms of $\Sigma_{m}$ we can, therefore, when $m \neq 2$ and $m \neq 6$, multiply $\tau$ by such an inner automorphism that the isomorphism $\alpha$ between $S_{m}$ and $S_{m}^{\top}$ is given by the relations (19).

Next let the $T_{s}^{(i)}$ in (15) denote a set of endomorphisms of $H$ satisfying the permutability conditions (12). The effect of any automorphism $\beta$ of $V_{m}$ upon any element $\eta$ in $V_{m}$ given by (13) is then expressed in (14), where it must be additionally verified that the $\beta$ thus defined is an automorphism and not only an endomorphism.

After having defined the correspondences $\alpha$ through (19) and $\beta$ through (14), one can find all automorphisms of $\Sigma_{m}$ through the combination of them in the manner indicated in the preceding. It remains only to verify when the conditions corresponding to (17) are satisfied for the two correspondences. One finds easily that it is sufficient to consider these conditions for generating elements of the two groups, hence for $\mu=(1, i), \nu=\eta_{j}$ where $\eta_{j}$ is defined by (10).

From

$$
(1, i) \eta_{j}(1, i)^{-1}=\eta_{j}, \quad i \neq j, j \neq 1,
$$

follows first

$$
\begin{aligned}
{\left[1, a_{2, i}, \cdots, a_{m, i}\right](1, i)\left[h_{j}^{T_{1}^{(i)}}, \cdots, h_{j}^{T_{m}^{(j)}}\right](1, i)\left[1, a_{2, i}^{-1}, \cdots, a_{m, i}^{-1}\right] } & \\
& =\left[h_{j}^{T_{1}^{(i)}}, \cdots, h_{j}^{T_{m}^{(j)}}\right] .
\end{aligned}
$$

After simplification this leads to the relations

$$
\begin{aligned}
h^{T_{i}^{(j)}} & =h^{T_{1}^{(i)}}, \\
a_{k, i} h_{k}^{T_{k}^{(i)}} a_{k, i}^{-1} & =h^{T_{k}^{(j)}}, \quad(k=1,2, \cdots, m)
\end{aligned}
$$

which hold for an arbitrary $h$ in $H$. The first relation (21) shows that if one puts

$$
T^{(j)}=T_{j}^{(j)}, \quad R^{(j)}=T_{i}^{(j)},
$$$$
i \neq j
$$

then one has the simpler form for the automorphism $\beta$ when $j \neq 1$ :

$$
\eta_{j}^{\beta}=\left[h_{j}^{R^{(j)}}, \cdots, h_{j}^{R^{(j)}}, h_{j}^{T_{j}^{(j)}}, h_{j}^{R^{(j)}}, \cdots, h_{j}^{R^{(j)}}\right] .
$$

With the notations (22) the second relation (21) splits into the two

$$
\begin{aligned}
& a_{k, i} h^{R^{(j)}} a_{k, i}^{-1}=h^{R^{(j)}}, \\
& a_{j, i} h^{T^{(j)}} a_{j, i}^{-1}=h^{T^{(j)}} .
\end{aligned}
$$

$\left(^{3}\right)$ O. Hölder, Bildung zusammengesetzter Gruppen, Mathematische Annalen, vol. 46 (1895), pp 321-422. 
Next we turn to the relation corresponding to (20) for $j=1$ or $j=i$,

$$
(1, i) \eta_{1}(1, i)=\eta_{j}
$$

This leads by (17) to the following three relations deduced in the same manner as (21)

$$
h^{T_{i}^{(1)}}=h^{R^{(i)}}, \quad h^{T_{1}^{(1)}}=h^{T^{(i)}}, \quad h^{T_{k}^{(1)}}=a_{k, i} h^{R^{(i)}} a_{k, i}^{-1} .
$$

From the second of these relations it follows that

$$
T=T_{1}^{(1)}=T^{(i)}
$$

is independent of $i$. From the first relation (25) one finds supplementing (23),

$$
\eta_{1}^{\beta}=\left[h_{1}^{T}, h_{1}^{R^{(2)}}, \cdots, h_{1}^{R^{(m)}}\right]
$$

and by combination of (23) and (27) one finds for the general element $\eta$ in $V_{m}$ defined in (13)

$$
\begin{aligned}
& \eta^{\beta}=\left[h_{1}^{T} h_{2}^{R^{(2)}} \cdots h_{m}^{R^{(m)}}, h_{1}^{R^{(2)}} h_{2}^{T} h_{3}^{R^{(3)}} \cdots h_{m}^{R^{(m)}},\right. \\
& \left.\cdots, h_{1}^{R^{(m)}} h_{2}^{R^{(2)}} \cdots h_{m-1}^{R^{(m-1)}} h_{m}^{T}\right] .
\end{aligned}
$$

By combining the first and the last relations (25) one also has

$$
h^{R^{(i)}}=a_{i, j} h^{R^{(j)}} a_{i, j}^{-1}
$$

and according to (24) $a_{i, j}$ is permutable with any $h^{T}$ and any $h^{R^{(k)}}$ if $i \neq k, j \neq k$.

After having derived the conditions corresponding to (17) it remains only to verify that the correspondence $\beta$ defined by (23) and (27) or (28) actually is an automorphism of the basis group. We observe first that the permutability conditions $(12)$ in this case became

$$
\begin{aligned}
h^{T} k^{R^{(i)}} & =k^{R^{(i)}} h^{T}, \\
h^{R^{(i)}} k^{R^{(j)}} & =k^{R^{(j)}} h^{R^{(i)}},
\end{aligned}
$$

When these relations hold the correspondence $\beta$ is an endomorphism according to Theorem 3. To make it an automorphism the relations

$$
\begin{aligned}
& h_{1}^{T} h_{2}^{R^{(2)}} h_{3}^{R^{(3)}} \cdots h_{m}^{R^{(m)}}=k_{1}, \\
& h_{1}^{R^{(2)}} h_{2}^{T} h_{3}^{R^{(3)}} \cdots h_{m}^{R^{(m)}}=k_{2}, \\
& h_{1}^{R^{(m)}} h_{2}^{R^{(2)}} \cdots h_{m-1}^{R^{(m-1)}} h_{m}^{T}=k_{m}
\end{aligned}
$$

must have a unique set of solutions $h_{i}$ for any set of elements $k_{i}$ in $H$. To 
analyze these conditions we put

$$
h_{i}=y_{i} h_{1}, \quad k_{i}=x_{i} k_{1}
$$

and form the quotient between the first and the $i$ th relation (31). Using the permutability conditions (30) this leads to the $m-1$ relations

$$
y_{i}^{T}\left(y_{i}^{-1}\right)^{R^{(i)}}=x_{i} \text {. }
$$

If one puts symbolically

$$
h^{T-R^{(i)}}=h^{T}\left(\dot{h}^{-1}\right)^{R^{(i)}},
$$

then $T-R^{(i)}$ is a correspondence of $H$ to a subset of itself. It should be noted that this correspondence need not be an endomorphism. When the notation (34) is used one can write (33) slightly simpler,

$$
y_{i}^{T-R^{(i)}}=x_{i} \quad(i=2, \cdots, m) .
$$

Since these conditions must be satisfied by some $y_{i}$ for every set of elements $x_{i}$ in $H$, it follows that $T-R^{(i)}$ is a one-to-one correspondence of $H$ to itself and conversely if this is the case the $y_{i}$ are uniquely determined by the $x_{i}$.

When (32) is substituted in the relations (31) it follows by the permutability conditions (30) and from (35) that the relations (31) reduce to a single condition

$$
y_{2}^{R^{(2)}} \cdots y_{m}^{R^{(m)}} h_{1}^{T} h_{1}^{R^{(2)}} \cdots h_{1}^{R^{(m)}}=k_{1} .
$$

Here it is convenient to put

$$
h^{S}=h^{T+R^{(2)}+\cdots+R^{(m)}}=h^{T} h^{R^{(2)}} \cdots h^{R^{(m)}},
$$

and it is seen immediately that

$$
S=T+R^{(2)}+\cdots+R^{(m)}
$$

is an endomorphism of $H$. If, however, there shall exist a unique solution $h_{1}$ of (36) for given $k_{1}$ and $y_{2}, \cdots, y_{m}$ it is seen that $S$ must be an automorphism of $H$. Conversely if this is the case and if all $T-R^{(i)}$ are one-to-one correspondences of $H$ to itself, the correspondence $\beta$ is an automorphism of the basis group. This concludes the set of conditions for an automorphism of $\Sigma_{m}$.

4. Explicit determination of the automorphisms. Through an analysis of the previous necessary and sufficient conditions for an automorphism one can obtain an explicit representation of all automorphisms of the symmetry.

Let us observe first that since the correspondence $T-R^{(i)}$ is a one-to-one correspondence of $H$ to itself, every element $\bar{h}$ in $H$ can be written in the form

$$
\bar{h}=h^{T}\left(h^{-1}\right)^{R^{(n)}}
$$

where $h$ is some element in $H$. Let $H^{T}$ and $H^{R^{(i)}}$ denote the subgroups on 
which $H$ is mapped by these endomorphisms. Then one concludes from (37) that

$$
H=H^{T} \cup H^{R^{(i)}} .
$$

But if $i \neq j$ then the elements of $R^{(j)}$ are permutable with the elements of $H^{R^{(i)}}$ and $H^{T}$ hence with all elements of $H$. This shows that $H^{R^{(i)}}$ belongs to the center of $H$. But since $R^{(i)}$ and $R^{(j)}$ differ only by an inner automorphism they must be identical and

$$
R=R^{(i)}
$$

does not depend on $i$, and one can write

$$
H=H^{T} \cup H^{R} \text {. }
$$

The elements $a_{i j}$ are permutable with the elements of $H^{T}$ and also with the elements of $H^{R}$ since they belong to the center.

This shows that also all $a_{i j}$ belong to the center. But the $a_{i j}$ are known to generate a group $A$ homomorphic to the symmetric group on $m-1$ letters. Thus one has only the two possibilities

$$
A \simeq 1, \quad A \simeq S_{m-1} / A_{m-1} .
$$

In the first case $a_{i j}=1$ for all $i$ and $j$. In the second case $A$ is cyclic of order 2 . Thus one can put in both cases

$$
a_{i j}=a, \quad i \neq j, \quad a^{2}=1
$$

where $a$ belongs to the center of $H$.

The endomorphism $R$ projects $H$ upon a subgroup of its center. One sees that such an endomorphism can only exist if the anti-center, i.e., the quotient group of the commutator group of $H$ has a subgroup isomorphic to a subgroup of the center. One sees also that if $R$ has this property and if the $a_{i j}$ are defined through (39) then all the preceding conditions are satisfied except that it remains to verify that the correspondence $\beta$ in (28) actually is an automorphism.

Under the conditions just derived the relation (28) simplifies to

$$
\eta \eta^{\beta}=\left[h_{1}^{S} p^{R}, h_{2}^{S} p^{R}, \cdots, h_{m}^{S} p^{R}\right]
$$

where we have put

$$
S=T-R
$$

and

$$
p=h_{1} h_{2} \cdots h_{m} \text {. }
$$

It should be noted that since $H^{R}$ belongs to the center the correspondence $S$ in (41) is actually an automorphism of $H$. 
The correspondence (40) can obviously be considered the product of two correspondences of the form

$$
\begin{aligned}
\eta^{\sigma} & =\left[h_{1}^{s}, h_{2}^{s}, \cdots, h_{m}^{s}\right], \\
\eta^{\kappa} & =\left[h_{1} p^{K}, h_{2} p^{K}, \cdots, h_{m} p^{K}\right]
\end{aligned}
$$

where $S$ and $p$ are defined by (41) and (42) while $K=R S^{-1}$ is also an endomorphism projecting $H$ upon a subgroup of the center. Since $\sigma$ is an automorphism of $V_{m}$ the correspondence $\beta$ is an automorphism if and only if $\kappa$ has this property. By a previous method one sees that this is the case if and only if the correspondence

$$
h \rightarrow h\left(h^{m}\right)^{K}=h^{1+m K}
$$

is an automorphism of $H$. This last automorphism is a central automorphism where every element is multiplied by an element in the center.

We have thus finally arrived at the main result:

TheOREM 4. Let $m \neq 2$ and $m \neq 6$. Any automorphism of the symmetry $\Sigma_{m}(H)$ can be obtained by transformation from an automorphism $\tau$ constructed in the following manner:

The images of the permutations in $\Sigma_{m}$ are determined by

$$
\begin{aligned}
(1, i)^{r} & =[1, a, \cdots, a, 1, a, \cdots, a](1, i), \\
(i, j)^{r} & =[a, \cdots, a](i, j),
\end{aligned} \quad i \neq 1, j \neq 1,
$$

where $a$ is any element of order 2 belonging to the center of $H$. The image of an element in the basis group

$$
\eta=\left[h_{1}, h_{2}, \cdots, h_{m}\right]
$$

is

$$
\eta^{\tau}=\left[\left(h_{1} p^{K}\right)^{S},\left(h_{2} p^{K}\right)^{S}, \cdots,\left(h_{m} p^{K}\right)^{S}\right]
$$

where $S$ is any automorphism of $H$ and

$$
p=h_{1} h_{2} \cdots h_{m},
$$

while $K$ is any endomorphism of $H$ projecting $H$ upon a subgroup of its center in such a manner that $1+m K$ is a central automorphism of $H$.

From this result all outer automorphisms of $\Sigma_{m}(H)$ can be determined. Let us find the conditions for $\Sigma_{m}(H)$ to be a complete group, i.e., a group in which all automorphisms are inner automorphisms. First it is easily observed that the correspondence determined in Theorem 4 for the permutations in $\Sigma_{m}$ cannot be obtained by an inner automorphism except when $a=1$. Thus the center of $H$ can contain no elements of order 2. When $a=1$ all permutations in $\Sigma_{m}$ are left invariant by $\tau$ and the only inner automorphisms in $\Sigma_{m}$ which 
will have this property are the scalars $[h]$. Next let us choose $K$ such that $h^{K}=1$ for every $h$ in $H$. Then one finds

$$
\eta^{\tau}=\left[h_{1}^{S}, \cdots, h_{m}^{S}\right]
$$

and this can only be obtained by transformation with a scalar if $S$ is an inner automorphism of $H$. Finally a correspondence

$$
\eta^{\tau}=\left[h_{1} p^{K}, \cdots, h_{m} p^{K}\right]
$$

is never obtainable by transformation with a scalar except when $h^{K}=1$ for every $h$. Thus we have shown:

THEOREM 5. Let $m \neq 2$ and $m \neq 6$. The necessary and sufficient condition for the symmetry $\Sigma_{m}(H)$ to be complete is that $H$ be complete and have a center of odd order. Furthermore, there shall exist no endomorphism $K$ of $H$ such that $H^{K}$ belongs to the center and $1+m K$ is an automorphism of $H$.

If $H$ has no center $\Sigma_{m}(H)$ is complete if and only if $H$ is complete.

Theorems 4 and 5 give the main results on the automorphisms of the symmetry when $m \neq 2$ and $m \neq 6$. The case $m=2$ will be considered in the following. The case $m=6$ is complicated by the fact that in this case the group $S_{m}$ has an outer automorphism. But since this automorphism is known $\left({ }^{4}\right)$, the preceding method can be used to determine the automorphisms of $\Sigma_{m}$ also in this case. Since the calculations are somewhat laborious they have not been carried through here. It would be of interest, if someone would take the trouble of carrying through this investigation.

5. Automorphisms for $m=2$. We shall now analyze the automorphisms of the symmetry in the exceptional case $m=2$. As we have already observed in $\$ 1$, the basis group $V_{2}(H)$ need not be invariant by the automorphisms of $\Sigma_{2}(H)$. We shall say, however, that an automorphism is regular if it does have the property that it leaves $V_{2}(H)$ invariant. We shall show a little later that except for a special type of groups $H$ the automorphisms of $\Sigma_{2}(H)$ are always regular.

We shall first determine the form of the regular automorphisms of $\Sigma_{2}(H)$ by the same method as the one used in $\$ 3$ in the general case. According to Theorem 2, Chapter I, the symmetry splits regularly over its basis group and it can be assumed, therefore, corresponding to (19) that we have multiplied the regular automorphism $\tau$ in question by an inner automorphism such that

$$
(1,2)^{\tau}=(1,2) \text {. }
$$

(4) According to O. Hölder (loc. cit.) the single outer automorphism to be considered may be taken as

$$
\begin{gathered}
(1,2) \rightarrow(1,2)(3,4)(5,6), \quad(1,3) \rightarrow(1,6)(2,3)(4,5), \quad(1,4) \rightarrow(1,5)(2,4)(3,6), \\
(1,5) \rightarrow(1,3)(2,5)(4,6), \quad(1,6) \rightarrow(1,4)(2,6)(3,5) .
\end{gathered}
$$


Furthermore one must have

$$
\left[h_{1}, 1\right]^{\tau}=\left[h_{1}^{T_{1}^{(1)}}, h_{1}^{T_{2}^{(1)}}\right]\left[1, h_{2}\right]^{\tau}=\left[h_{2}^{T_{1}^{(2)}}, h_{2}^{T_{2}^{(2)}}\right]
$$

with the permutability conditions (12). By taking the image of the relation

$$
(1,2)[h, 1](1,2)=[1, h]
$$

one obtains the conditions

$$
T=T_{1}^{(1)}=T_{2}^{(2)}, \quad R=T_{2}^{(1)}=T_{1}^{(2)}
$$

and consequently

$$
\left[h_{1}, h_{2}\right]^{\top}=\left[h_{1}^{T} h_{2}^{R}, h_{2}^{T} h_{1}^{R}\right]
$$

where the elements of the two groups $H^{T}$ and $H^{R}$ are permutable. It remains only to determine when this homomorphism of $V_{2}(H)$ is an automorphism. One finds that the relations

$$
\stackrel{T}{h_{1}} h_{2}^{R}=k_{1}, \quad \stackrel{T}{h}_{2}^{R} h_{1}^{R}=k_{2}
$$

can be solved uniquely for $h_{1}$ and $h_{2}$ with arbitrary $k_{1}$ and $k_{2}$ in $H$ if and only if $T-R$ is a one-to-one correspondence of $H$ to itself while $T+R$ is an automorphism of $H$. Thus we have shown:

THEOREM 6. For $m=2$ a regular automorphism of $\Sigma_{2}(H)$ differs only by an inner automorphism from the automorphism defined by

$$
\begin{aligned}
(1,2)^{\top} & =(1,2), \\
{\left[h_{1}, h_{2}\right]^{\tau} } & =\left[h_{1}^{T} h_{2}^{R}, h_{2}^{T} h_{1}^{R}\right]
\end{aligned}
$$

where the endomorphisms $T$ and $R$ of $H$ have the property that

$$
h_{1}^{T} h_{2}^{R}=h_{2}^{R} h_{1}^{T}
$$

and $T+R$ is an automorphism of $H$ while $T-R$ is a one-to-one correspondence of $H$ to itself.

The existence of two such endomorphisms $T$ and $R$ of $H$ implies a permutable decomposition

$$
H=H^{T} \cup H^{R} .
$$

Conversely if a permutable decomposition of $H$ exists then one can obtain by a few additional conditions that endomorphisms $T$ and $R$ with the desired properties will exist. We shall not discuss these conditions here.

We shall now determine when irregular automorphisms of $\Sigma_{2}(H)$ can exist. For an irregular automorphism $\tau$ there must exist some subgroup $\bar{M}$ such that 


$$
\bar{M}^{\tau}=V_{2}(H) \text {. }
$$

Here $\bar{M}$ must be normal and obviously

$$
\Sigma_{2}(H)=\bar{M} \cup V_{2}(H) .
$$

Since $\bar{M}$ is isomorphic to $V_{2}$ one can write

$$
\bar{M}=\bar{H} \times \overline{\bar{H}}
$$

where each direct component is isomorphic to $H$. The group $\bar{M}$ must contain cycles

$$
\gamma=\left(\begin{array}{ll}
x_{1}, & x_{2} \\
a x_{2}, & b x_{1}
\end{array}\right)
$$

and consequently at least one of the groups $\bar{H}$ and $\overline{\bar{H}}$ contains such cycles. Since $\bar{M}$ is normal in $\Sigma_{2}$ and since any transformation of $\bar{M}$ results in some other decomposition (43) we can assume that $\bar{H}$ contains a cycle

$$
\gamma_{0}=\left(\begin{array}{l}
x_{1}, x_{2} \\
x_{2}, a x_{1}
\end{array}\right)
$$

in the normal form.

The centralizer of such a cycle $(45)$ has already been determined in $\$ 4$, Chapter I and it was found to consist of the elements of the form

$$
\gamma_{0}^{i}[c, c] \quad(i=0,1)
$$

where $c$ is any element in the centralizer of $a$ in $H$.

The order of the centralizer of $\gamma_{0}$ in $\Sigma_{2}$ is, therefore, $2 c_{a}$ where $c_{a}$ is the order of the centralizer of $a$ in $H$. On the other hand, all elements in $\bar{H}$ must belong to the centralizer of $\gamma_{0}$ according to the direct decomposition (43). Furthermore some elements of $\bar{H}$, for instance the powers of $\gamma_{0}$ must belong to it. Thus the order of the centralizer becomes at least $n_{0} \cdot n_{H}$ where $n_{0}$ is the order of $\gamma_{0}$ and $n_{H}$ the order of $H$. By comparison with the previous determination of the order of the centralizer this can only be possible if $c_{a}=n_{H}$ and $n_{0}=2$. But then

$$
\gamma_{0}^{2}=[a, a]=1
$$

and $a=1$, so that $\gamma_{0}$ in (45) takes the special form $\gamma_{0}=\left(x_{1}, x_{2}\right)$. It has been shown, therefore, that every cycle $\gamma$ in (44) contained in $\bar{H}$ has the order 2 and consequently it has the special form

$$
\gamma=\left(\begin{array}{ll}
x_{1}, & x_{2} \\
a x_{2}, & a^{-1} x_{1}
\end{array}\right) .
$$

Now let us assume again that $\bar{H}$ has been so transformed that it contains 
$\gamma_{0}=\left(x_{1}, x_{2}\right)$. We expand $\bar{H}$ in cosets

$$
\bar{H}=\bar{H}_{0}+\gamma_{0} \bar{H}_{0}
$$

where $\bar{H}_{0}$ is the normal subgroup of $\bar{H}$ consisting of its multiplications. If $[a, b]$ is an element of $\bar{H}_{0}$ then $\gamma_{0}[a, b]$ is a cycle contained in $\bar{H}$ and from the preceding result it follows that $b=a^{-1}$. Thus all the elements of $\bar{H}_{0}$ have the form

$$
\alpha=\left[a, a^{-1}\right]
$$

If

$$
\beta=\left[b, b^{-1}\right]
$$

is another element in $\bar{H}_{0}$ then their product

$$
\alpha \beta=\left[b a, b^{-1} a^{-1}\right]=\left[b a,(b a)^{-1}\right]
$$

is also in $\bar{H}_{0}$. This implies $a b=b a$ and $\bar{H}_{0}$ is Abelian. Since $\bar{H}$ is isomorphic to $H$ this shows that $H$ is obtained from an Abelian group $H_{0}$ by an extension of order 2.

One can make a further statement about the group $H_{0}$. It follows from the preceding that the group $\bar{H}$ cannot contain any other elements of the centralizer of $\gamma_{0}$ than 1 and $\gamma_{0}$ itself. However all elements $[a, a]$ belong to the centralizer of $\gamma_{0}$ and such elements must be contained in $\bar{H}$ if $\bar{H}_{0}$ contains an element $\left[a, a^{-1}\right]$ with $a^{2}=1$. This occurs if and only if $\bar{H}_{0}$ contains elements of order 2 . Hence we have shown:

THEOREM 7. Irregular automorphisms of $\Sigma_{2}(H)$ can only exist if $H$ contains a normal Abelian subgroup $H_{0}$ of odd order and index 2.

If $H$ has the form indicated in Theorem 7 one can write

$$
H=A \cup H_{0}, \quad A \cap H_{0}=E
$$

where

$$
A=\{a\}, \quad a^{2}=1
$$

is a cyclic group of order 2 . Let us write $V_{2}\left(H_{0}\right)$ for the group consisting of all elements

$$
\left[h_{1}^{(0)}, h_{2}^{(0)}\right]
$$

where $h_{1}^{(0)}$ and $h_{2}^{(0)}$ run through $H_{0}$ independently. Obviously $V_{2}\left(H_{0}\right)$ is a normal subgroup of $\Sigma_{2}(H)$. Since its order is an odd number it is relatively prime to its index 8 , consequently $V_{2}\left(H_{0}\right)$ is a characteristic subgroup. It is also seen that $\Sigma_{2}(H)$ splits over $V_{2}\left(H_{0}\right)$ :

$$
\Sigma_{2}(H)=\Sigma_{2}(A) \cup V_{2}\left(H_{0}\right), \quad \Sigma_{2}(A) \cap V_{2}\left(H_{0}\right)=E
$$


where $\Sigma_{2}(A)$ is the group of order (8) whose elements are given in (5). We have shown that $\Sigma_{2}(A)$ was the simplest group for which irregular automorphisms existed.

The automorphisms of $\Sigma_{2}(H)$ can now be constructed by the method indicated in $\$ 3$ from the decomposition (46). The possible automorphisms of $V_{2}\left(H_{0}\right)$ are defined by

$$
[k, 1]^{\tau}=\left[k_{1}^{T_{1}^{(1)}}, k_{2}^{T_{2}^{(1)}}\right]\left[1, k_{2}\right]^{\tau}=\left[k_{2}^{T_{1}^{(2)}}, k_{2}^{T_{2}^{(2)}}\right]
$$

where $k_{1}$ and $k_{2}$ are elements of $H_{0}$ and the $T$ denote endomorphisms of $H_{0}$ satisfying the permutability conditions (12).

Since the order of $V_{2}\left(H_{0}\right)$ is relatively prime to its order the group $\Sigma_{2}(H)$ splits regularly over $V_{2}\left(H_{0}\right)$, i.e., any other group which can replace $\Sigma_{2}(A)$ in (46) is obtained from $\Sigma_{2}(A)$ by an inner automorphism. Since $\tau$ may be multiplied by an arbitrary inner automorphism one can assume that $\tau$ leaves $\Sigma_{2}(A)$ invariant. This means that the 8 elements of $\Sigma_{2}(A)$ are transformed by $\tau$ according to the automorphism of $\Sigma_{2}(A)$ already determined in $\S 1$. The elements

$$
x=\left(x_{1}, x_{2}\right), \quad y=\left(x_{1}, x_{2}\right)[a, 1]
$$

generate the group and one has according to (7) and (9)

$$
x^{\tau}=[a, 1], \quad y^{\tau}=y .
$$

The method indicated in $\$ 3$ can now be used. We shall not go into the details of the calculations, but only give the final result:

THEOREM 8. Let $H$ be a group for which $\Sigma_{2}(H)$ can have irregular automorphisms. Then $H$ has a normal Abelian subgroup $H_{0}$ of odd order and of index 2, hence

$$
H=\{a\} \cup H_{0}, \quad a^{2}=1 .
$$

The irregular automorphisms of $\Sigma_{2}(H)$ are then determined by

$$
\begin{aligned}
\left(x_{1}, x_{2}\right)^{r} & =[a, 1], \\
{[a, 1]^{r} } & =\left(x_{1}, x_{2}\right)[a, a], \quad[1, a]^{r}=\left(x_{1}, x_{2}\right),
\end{aligned}
$$

and

$$
\left[h_{1}, h_{2}\right]^{T}=\left[\left(h_{1} h_{2}^{a}\right)^{T},\left(h_{1} h_{2}\right)^{T_{1}}\right], \quad h_{1}, h_{2} \text { in } H_{0},
$$

where $h^{a}=a h a^{-1}$ and $T$ denotes an automorphism of $H_{0}$ such that

$$
\left(h^{a}\right)^{T}=\left(h^{T}\right)^{a} .
$$

Furthermore the element a must satisfy the condition that every element in $H_{0}$ is expressible as a commutator $h a h^{-1} a^{-1}$. 


\section{Chapter IV. Monomial representations}

1. Construction of monomial representations. Let $G$ be some group. A monomial representation of $G$ is a homomorphism of $G$ to some subgroup $M_{G}$ of the symmetry $\Sigma_{m}(H)$ of some group $H$. A monomial representation may also be considered a homomorphism of $G$ to a set of matrices such that to each $g$ in $G$ there corresponds a monomial matrix $M_{\theta}$, i.e., a matrix of the special type that each line and column contains only one nonvanishing element. Since the multiplication of such matrices involves only the products of the elements which occur in them, one can suppose that the elements in the monomial matrices belong to some group $H$.

If $K$ is some normal subgroup of $H$ then any monomial representation in $H$ also gives a monomial representation in the quotient group $H / K$ simply by considering the elements of $H(\bmod K)$.

For the monomial groups one can introduce the analogues of the ordinary concepts of transitivity and primitivity. Any monomial representation can be written uniquely as a product of transitive representations.

All transitive monomial representations of a group can be obtained by the following construction:

Let $H$ be a subgroup of $G$ of finite index $m$ and let

$$
G=H g_{1}+\cdots+H g_{m},
$$$$
g_{1}=1,
$$

be the corresponding coset expansion. Then for any $z$ in $G$ one has a relation

$$
g_{i} z=h_{i}^{(z)} g_{z: i \text {. }}
$$

This relation defines for each $z$ a monomial substitution

$$
M_{z}=\left(\begin{array}{llll}
x_{1}, & x_{2}, & \cdots, & x_{m} \\
h_{1}^{(z)} & x_{2: 1}, & h_{2}^{(z)} & x_{z: 2}, \cdots, h_{m}^{(z)} \\
x_{z: m}
\end{array}\right) .
$$

The elements $h_{i}^{(z)}$ in $H$ shall be called the factors of $z$. If $t$ is another element in $G$ and

$$
g_{j} t=h_{j}^{(t)} g_{t: j}
$$

then

$$
g_{i}(z t)=h_{i}^{(z)} g_{z: i} t=h_{i}^{(z)} h_{z: i}^{(t)} g_{t z: i},
$$

and one sees that

$$
M_{2 t}=M_{t} M_{2} .
$$

This shows that there exists a transposed homomorphism between the elements in $G$ and the monomial substitutions (3). One can of course obtain a direct homomorphism by letting $M_{z}$ in (3) correspond to the element $z^{-1}$. 
If $K$ is any normal subgroup of $H$ then one obtains a monomial representation of $G$ with respect to $H / K$ by considering the factors $h_{\imath}^{(z)}$ in $(3)(\bmod K)$.

From the manner in which the construction of the monomial representation has been performed it follows that there exist substitutions $M_{0}$ taking an arbitrary variable $x_{i}$ into any $h x_{j}$. The elements in $H$ correspond to substitutions for which $x_{1} \rightarrow h x_{1}$ and those in $K$ to substitutions in which $x_{1} \rightarrow x_{1}$.

Conversely it is easily seen that all transitive monomial representations may be obtained in this manner. For a given monomial group, the group $H$ consists of those substitutions for which $x_{1} \rightarrow h_{1} x_{1}$ and $K$ those for which $x_{1} \rightarrow x_{1}$, while the generator $g_{i}$ in (1) is taken as the substitution for which $x_{1} \rightarrow x_{i}$.

The preceding theory applies only to a group $G$ having a subgroup of finite index. There is however no serious difficulty in extending the theory to the case of an arbitrary transfinite number of cosets in (1). This would involve the extension of the theory of the symmetry $\Sigma_{m}(H)$ to such transfinite deg rees.

The question immediately arises when the monomial representation is isomorphic to the group $G$. If an element $z$ shall correspond to the unit substitution, one must have, according to (2), $g_{i} z g_{i}^{-1} \subset K$ for all $i$, hence $K$ contains a normal subgroup of $G$. Conversely all the elements of such a subgroup of $K$ must correspond to the unit substitution.

We may summarize these results as follows:

TheOREM 1. All transitive monomial representations of a group $G$ are obtainable by selecting a subgroup $H$ of $G$ and a normal subgroup $K$ of $H$ and constructing the monomial representation of $G$ in $H / K$ by the coset expansion (1) and defining the factors of the substitutions from (2). The corresponding representation is isomorphic to $G / N$ where $N$ is the largest normal subgroup of $G$ contained in $K\left({ }^{5}\right)$.

2. Various properties of the monomial representations. We observe first that the monomial representation of a group obtained by the process just in: dicated depends on the choice of the representatives $g_{i}$ in the coset expansion (1). If $g_{i}$ is replaced by

then one finds from $(2$;

$$
\bar{g}_{i}=\bar{h}_{i} g_{i}
$$

$$
\bar{g}_{i} z=\bar{h}_{i} g_{i} z=\bar{h}_{i} h_{i}^{(z)} g_{z: i}=\bar{h}_{i} h_{i}^{(z)} \bar{h}_{z: i}^{-1} \bar{g}_{z: i},
$$

hence the factors in the new representation are

$$
f_{i}=\bar{h}_{i} h_{i}^{(z)} \bar{h}_{z: i}^{-1} \text {. }
$$

But this shows that $M_{2}$ is replaced by the substitution

(b) See W. K. Turkin, loc. cit. 


$$
\bar{M}_{z}=\kappa^{-1} M_{z} \kappa
$$

where $\kappa$ is the multiplication

$$
\kappa=\left[\bar{h}_{1}, \cdots, \bar{h}_{m}\right] .
$$

One could also have changed the order of the cosets in (1) and this would have the effect of renumbering the variables $x_{i}$. But any such change can also be obtained by transforming $M_{z}$ by a permutation and we can therefore say:

THEOREM 2. All the different transitive monomial representations of a group $G$ in quotient group $H / K$ which can be obtained through permutations of cosets and change of representatives are conjugate subgroups of $\Sigma_{m}(H / K)$. Conversely any two transitive conjugate subgroups of $\Sigma_{m}(H / K)$ can be considered as representatives of the same group where one representation is obtained from the other by the two processes indicated.

The actual determination of the monomial substitution corresponding to a given element $z$ may of ten be made by such a transformation. One chooses the representatives in the coset expansion (1) in sets

$$
g_{i}, g_{i} z, \cdots, g_{i} z^{f_{i}-1}
$$

where $f_{i}$ is the smallest exponent such that

$$
g_{i} z^{f_{i}} \subset H g_{i}
$$

or

$$
z^{f_{i}} \subset g_{i}^{-1} H g_{i}
$$

If one writes

$$
g_{i} z^{i}=d_{i} g_{i}
$$

then one finds that in the monomial representation $z \rightarrow M_{z}$ where $M_{z}$ is in the normal form

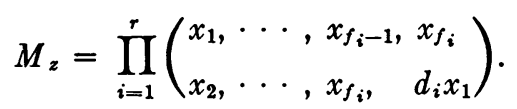

The cycles in this substitution correspond to the terms in the expansion of the group into double cosets

$$
G=H Z+H g_{2} Z+\cdots+H g_{m} Z
$$

where $Z=\{z\}$ is the cyclic group defined by $z$. Each double coset in ( 7$)$ contains $f_{i}$ cosets with respect to $H$ and

$$
\sum_{i=1}^{r} f_{i}=m
$$


These remarks permit us to decide when the substitution $M_{z}$ corresponding to $z$ can be transformed into a permutation. In this case one must have $d_{i}=1$ in (6) for every $i$ and one concludes:

THEOREM 3. Let $z$ be an element in $G$ and $M_{z}$ the monomial substitution corresponding to $z$ in the representation of $G$ with respect to a quotient group $H / K$. Then a necessary and sufficient condition that $M_{z}$ be transformable into a permutation is that the elements of the cyclic group $Z=\{z\}$ and its conjugates which belong to $H$ also belong to $K$.

One can also find when the monomial substitution corresponding to $z$ is a multiplication. In this case one must have, for every $g_{i}$ in (1),

$$
g_{i} z=h_{i}^{(z)} g_{i}
$$

or

$$
g_{i} z g_{i}^{-1} \subset H
$$

hence the element $z$ must belong to a normal subgroup of $G$ contained in $H$. Conversely if $z$ belongs to such a group the relation (9) will hold.

THEOREM 4. Those elements of $G$ which correspond to multiplications in the monomial representation of $G$ with respect to $H / K$ form a group which is the greatest normal subgroup of $G$ contained in $H$.

Let us consider briefly the case when $H$ is normal in $G$. Then every element in $G$ has the form $z=h g_{j}$. The monomial substitution corresponding to $h$ is determined by

$$
g_{i} h=h^{\prime} g_{i}, \quad h^{\prime}=h^{o_{i}},
$$

hence $M_{h}$ is the multiplication

$$
M_{h}=\left[h^{o_{1}}, \cdots, h^{o_{m}}\right] .
$$

Corresponding to the representative $g_{j}$ in the coset expansion one has

$$
g_{i} g_{j}=f_{i, j} g_{i, j}
$$

where the $f_{i, j}$ form the factor set of the extension $G$ over $H$, hence

$$
M_{u_{j}}=\left(\begin{array}{l}
x_{1}, \cdots, x_{m} \\
f_{1, j} x_{1, j}, \cdots, f_{m, j} x_{m, j}
\end{array}\right) .
$$

3. The normalizer of a subgroup in the symmetry. Let $G$ be a group represented monomially with respect to a quotient group $H / K$. As a subgroup of the complete monomial group $\Sigma_{m}(H / K)$ the group $G$ will have a certain normalizer $N_{G}$. We shall now study the properties of those automorphisms which the elements of $N_{G}$ induce in the group $G$. 
Let $\nu$ be an element of $N_{G}$ taking the variable $x_{1}$ into $x_{i}$. One sees then that

$$
\nu H \nu^{-1}=H_{i}
$$

where $H_{i}$ is the subgroup of $G$ consisting of those substitutions multiplying $x_{i}$ by a factor. This shows that $\nu$ transforms all groups $H_{i}$ among themselves.

Now there also exists some inner automorphism $g$ of $G$ such that

$$
g H g^{-1}=H_{i}
$$

and one finds

$$
\mu H \mu^{-1}=H, \quad \mu=\nu g^{-1} .
$$

One needs therefore only to study substitutions $\mu$ transforming $H$ into itself. But if

$$
\mu=\left(\begin{array}{ll}
x_{1}, & \cdots \\
h_{1} x_{1}, & \ldots
\end{array}\right)
$$

one can multiply $\mu$ further by such an element in $M_{H}$ that $h_{1}=1$. In this case one sees that

$$
\mu H \mu^{-1}=H, \quad \mu K \mu^{-1}=K
$$

and further by transformation with $\mu$ the quotient group $H / K$ is left elementwise invariant.

Conversely let $A$ be an automorphism of $G$ leaving $H / K$ elementwise invariant. Then one can associate with $A$ a monomial substitution

$$
\mu=\left(\begin{array}{ll}
\cdots, x_{i}, & \cdots \\
\cdots, t_{i} x_{A: i}, & \cdots
\end{array}\right)
$$

where the factors $t_{i}$ are defined by

$$
\stackrel{A}{g_{i}}=t_{i} g_{A: i}
$$

Next we form the substitution

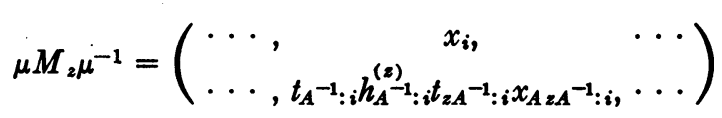

for an arbitrary element $z$ in $G$. We shall show that

$$
\mu M_{z} \mu^{-1}=M_{z} A \text {. }
$$

To construct the factors of $M_{2} A$ we observe that

$$
g_{i} z^{A}=t_{A^{-1}: i}^{-1}\left(g_{A^{-1}: i} z\right)^{A}=t_{A^{-1}: i}^{-1}\left(h_{\left.A^{-1}: i g_{z A^{-1}: i}\right)^{A}}^{(z)}\right.
$$

and since $A$ leaves $H / K$ invariant 


$$
g_{i} z^{A}=t_{A^{-1}: i} h_{A}^{(z)}: i t_{2 A^{-1}: i g_{A z A^{-1}: i}}
$$

as desired. We have proved therefore:

THEOREM 5. Let $G$ be a group which is represented as a transitive monomial group of degree $m$ with respect to a quotient group $H / K$. Then the normalizer $N_{G}$ of $G$ in the symmetry $\Sigma_{m}(H / K)$ consists of all those substitutions which aside from a factor in $G$ induce such automorphisms in $G$ that the quotient group $H / K$ is left elementwise fixed.

Under certain circumstances all automorphisms of $G$ may be induced by the elements of the normalizer $N_{G}$. Let us determine when this occurs. We denote by $A$ an arbitrary automorphism of $G$. Then one must have

$$
H^{A}=H_{i}=H^{o_{i}}
$$

for some $g_{i}$ in $G$, hence $A$ has the effect of an inner automorphism upon $H$ and $H$ is a typical $\left(^{6}\right)$ subgroup of $G$. Then $B=A g_{i}^{-1}$ leaves $H$ invariant and

$$
K^{B}=K, \quad K^{A}=K^{g_{i}},
$$

hence $H$ and $K$ are compactible typical subgroups. Finally $H / K$ must be left elementwise invariant by $B$.

THEOREM 6. The necessary and sufficient condition that all automorphisms of $G$ shall be induced by the elements of the normalizer $N_{G}$ of $G$ in the symmetry $\Sigma_{m}(H / K)$ is that $H$ and $K$ be typical compatible subgroups of $G$ such that any automorphism of $G$ which leaves $H$ fixed, shall, aside from an inner automorphism of $H$, leave the quotient group $H / K$ elementwise fixed.

The conditions of this theorem are satisfied when:

1. $H$ is typical,

2. $K$ is characteristic in $H$,

3. $H / K$ is complete, i.e. all automorphisms are inner automorphisms.

The two first conditions are, for instance, always satisfied for a Sylow group and its normalizer.

4. The centralizer of a subgroup in the symmetry. Again let $G$ be a group represented as a monomial group with respect to a quotient group $H / K$. As a subgroup of $\Sigma_{m}(H / K)$ the group $G$ has a certain centralizer $Z_{G}$ whose properties we shall now study.

The center $\bar{C}$ of $\Sigma_{m}$ consists of the multiplications

$$
\mu=[c, \cdots, c]
$$

where $c$ belongs to the center of $H / K$. Obviously the center of $\Sigma_{m}$ belongs to $Z_{G}$. We can prove further:

${ }^{(6)}$ Such groups will be studied in greater detail in another paper on group theory which will be submitted for publication shortly. 
Any multiplication in $\Sigma_{m}$ belonging to $Z_{G}$ must belong to the center $\bar{C}$ of $\Sigma_{m}$.

Proof. Let the multiplication

$$
\mu=\left[c_{1}, \cdots, c_{n}\right]
$$

belong to $Z_{G}$. Since there exist elements in $G$ carrying $x_{i}$ into $h x_{i}$ for any $i$, where $h$ is an arbitrary element in $H / K$, one must have

$$
c_{i} h c_{i}^{-1}=h
$$

and $c_{i}$ must belong to the center of $H / K$. Furthermore since $G$ contains elements carrying $x_{1}$ into $x_{i}$ one finds $c_{1}=c_{i}$ for every $i$.

Next we show:

Let $\sigma$ be a substitution belonging to $Z_{G}$ which only changes some variable by a factor. Then $\sigma$ is a multiplication and belongs to the center of $\Sigma_{m}$.

Proof. Let $\sigma$ take $x_{i}$ into $h x_{i}$ and let $M_{z}$ be some substitution in $G$ carrying $x_{i}$ into some other variable $x_{j}$. Then one finds

$$
\sigma: x_{j}=\sigma M_{z}: x_{i}=M_{2} \sigma: x_{i}=M_{z}: h x_{i}=h x_{j}
$$

and $\sigma$ is a multiplication. This result also shows that two substitutions in $Z_{G}$ taking a variable $x_{i}$ into the same variable $x_{j}$ differ only by a factor which is an element of $\bar{C}$.

We shall now give a method of constructing elements of $\Sigma_{m}$ which belong to $Z_{G}$. Let $z$ be an element in $G$ which belongs to the normalizer of $H$ and in addition has the property that it leaves the quotient group $H / K$ elementwise invariant by transformation. Then one can associate with $z$ a unique monomial substitution

where

$$
S_{z}=\left(\begin{array}{ll}
\cdots, x_{i}, & \cdots \\
\cdots, t_{i} x_{8: i}, \cdots
\end{array}\right)
$$

$$
z g_{i}=t_{i} g_{s: i}
$$

Next let $y$ be an arbitrary element in $G$ and let us consider the product $z g_{i} y$. By the associative law this product, which belongs to $G$ can be evaluated in two ways, namely, first

$$
\left(z g_{i}\right) y=\left(t_{i} g_{s: i}\right) y=t_{i} h_{s: i}^{(y)} g_{y s: i},
$$

and secondly by the stated property of $z$

$$
z\left(g_{i} y\right)=z h_{i}^{(y)} g_{y: i}=h_{i}^{(y)} t_{y: i} g_{s y: i}
$$

This implies, however, $M_{y} S_{z}=S_{z} M_{y}$, hence $S_{z}$ belongs to $Z_{G}$. 
We have thus established a correspondence $z \rightarrow S_{z}$ between the elements $z$ of $G$ which leave $H / K$ elementwise invariant by transformation and certain elements $S_{z}$ of $Z_{G}$. This correspondence is seen to be a homomorphism and the elements corresponding to the unit element are the elements of $K$.

Now conversely let

$$
S=\left(\begin{array}{ll}
x_{1}, & \cdots \\
h_{1} x_{i}, & \cdots
\end{array}\right)
$$

be an element in $Z_{G}$. Then there exists an element $z$ in $G$ such that

$$
M_{z}=\left(\begin{array}{ll}
x_{1}, & \cdots \\
h_{1} x_{i}, & \cdots
\end{array}\right)
$$

and the element $z$ is determined uniquely $(\bmod K)$. Let $M_{h}$ be the substitution corresponding to an arbitrary element $h$ in $H$. Then

$$
M_{2} M_{h} M_{z}^{-1}: x_{1}=M_{z} M_{h} M_{z}^{-1} \cdot S^{-1} \cdot M_{2}: x_{1}=M_{2} S^{-1} M_{h}: x_{1}=M_{z} S^{-1}: h_{1} x_{1}=h_{1} x_{1} \text {. }
$$

This shows that

$$
M_{z} M_{h} M_{z}^{-1} \equiv M_{h}\left(\bmod M_{K}\right),
$$

hence $M_{z}$ and $z$ have the property of leaving $H / K$ invariant by transformation. By this correspondence between $S$ and $z$ the unit element corresponds to the elements in $K$. We have shown therefore:

Theorem 7. The centralizer $Z_{G}$ of a group $G$ in the symmetry $\Sigma_{m}(H / K)$ is isomorphic to the quotient group $M / K$ where $M$ consists of the elements in $G$ which have the property of leaving the quotient group $H / K$ elementwise invariant by transformation.

One may ask finally when the centralizer consists only of elements in $G$, hence when $Z_{G}$ is equal to the center of $G$. Let $z$ be an element in $G$ leaving $H / K$ invariant by transformation. The corresponding substitution $S_{z}$ belongs to $G$ if there exists an element $y$ in $G$ such that $z g_{i} \equiv g_{i} y(\bmod K)$. Clearly $y$ must belong to the center $C_{G}$ of $G$ and for $i=1$ one finds $z=y \cdot k$ where $k$ belongs to $K$. This gives immediately:

THEOREM 8. The necessary and sufficient condition that the centralizer of $G$ in $\Sigma_{m}(H / K)$ be the center $C_{G}$ of $G$ is that

$$
M=C_{G} \cup K
$$

where $M$ denotes the subgroup of $G$ whose elements have the property of leaving the quotient group $H / K$ elementwise invariant by transformation.

YALE UNIVERSITY,

New Haven, Conn. 